\title{
ON ORDINARY LINEAR $p$-ADIC DIFFERENTIAL EQUATIONS
}

\author{
BY
}

B. DWORK AND P. ROBBA

\begin{abstract}
We study the solutions of ordinary linear differential equations whose coefficients are analytic elements. As one application we show nonexistence of index for certain linear differential operators with rational function coefficients.
\end{abstract}

Introduction. This article represents a continuation of previous interdependent nonjoint work [3], [4], [11], [12], [13] on p-adic differential equations. This previous work was to a considerable extent restricted to the case of ordinary differential equations with rational coefficients. The main topics involved were

1. Growth of solutions at the boundary of disk of convergence.

2. Field of definition of the space of solutions having specified growth.

3. Existence of index.

The basic idea in this work has been the relation between solutions at the generic point and solutions at an arbitrary point. In particular, we have been concerned with the reducibility of differential operators corresponding to filtration of the solution space at the generic point as given by growth conditions.

We had at one time been of the opinion that a linear differential equation with rational function coefficients which is irreducible in that ring cannot have solutions at a generic point with distinct radii of convergence. We are indebted to Monsky for the counterexample ([11, §4.26.1], also $\$ 7$ below). This phenomenon is the main topic of the present article. Our main result (\$4.1) is that distinct radii of convergence of solutions does imply a strong form of reducibility (the factors need not have rational coefficients but do have superadmissible coefficients).

A natural illustration of this phenomenon is provided by the theory of elliptic modular functions if we view $y=j(p \tau)$ as a function of $x=j(\tau)$. The modular equation $F_{p}(x, y)$ is known to have solutions for $y$ with different radii

Received by the editors January 23, 1976.

AMS (MOS) subject classifications (1970). Primary 12B40, 34A25.

Key words and phrases. Nonarchimedean valuation, completion field, analytic element, analytic function, generic disk, differential equation. 
of convergence at the generic point. Since algebraic functions certainly satisfy a differential equation with rational coefficients we may conclude that "geometric" examples occur.

The clarification achieved on this question has permitted considerable improvement of earlier articles. For example (as noted in [11, §4.26.3]) we now know that if $L$ is a linear differential operator with rational coefficients, then for all but a finite number of residue classes $L$ has index as endomorphism of the space of functions analytic on a residue class. (For a more complete statement see $\$ 4.2$ below.) We are now able to remove the hypothesis of rational coefficients in the theory [3] of the growth at the circle of convergence of solutions of ordinary differential equations (cf. $\$ 4.2 .6$ below). In $\$ 7$ we use the example of Monsky to disprove earlier conjectures [3] concerning existence of index. In $\$ 6$ we give Hensel type lemmas for factorization of differential operators which lift factorizations of the reduced differential operators. The precise relation between the results of $\S 4$ and $\$ 6$ is not yet understood.

Our method has been to apply earlier results [11] concerning linear operators to obtain information concerning a general system of nonlinear differential equations, (3.1.6). The relation between nonlinear differential equations and reducibility of differential operators is discussed in $\$ 4.1$. Further progress (e.g. Conjecture 9.1.5 below) would result from improvements (such as Conjecture 3.1.5 below) in the theory of nonlinear differential operators.

\begin{tabular}{llcl} 
& \multicolumn{3}{c}{ Symbols } \\
$\mathfrak{Q}_{a}, \mathfrak{Q}_{a}^{\rho}$ & 1.7 & $\mathfrak{R}^{[m]}$ & 6.2 .2 \\
$\mathfrak{Q}_{\Delta}$ & 1.7 & $\bar{\Re}^{[m]}$ & 6.2 .2 \\
$\left(\mathfrak{Q}_{t}[D]\right)_{m}$ & 4.1 .3 & $\bar{\Re}_{0}$ & 6.1 \\
$D\left(a, b^{+}\right)$ & 1.2 & $\mathfrak{S}_{A}$ & 1.5 \\
$D\left(a, b^{-}\right)$ & 1.2 & $t$ & 1.1 \\
$E$ & 1.3 & $W_{A}$ & 1.12 \\
$E_{\mathrm{ad}}$ & 1.11 & $\dot{W}_{A}$ & 1.12 \\
$e_{A}$ & $1.9,1.12$ & $W_{A}^{\rho, \alpha}$ & 1.8 \\
$E_{0}$ & 1.1 & \|\|$_{\pi}$ & 1.6 \\
$\bar{E}_{0}$ & 6.1 & \|\|$_{\sigma}$ & 2.2 \\
$E_{\text {sad }}$ & 1.11 & \|\|$_{1,0}$ & 1.8 \\
||$_{E}$ & 1.3 & $\Omega$ & 1.1 \\
$|f|_{a}(r)$ & 1.2 & admissible $\left\{\begin{array}{l}\text { set } \\
\text { subset }\end{array}\right.$ & 1.11 \\
$H(A), H_{0}(A)$ & 1.5 & generic point & 1.1
\end{tabular}




$\begin{array}{llll}K & 1.1 & \begin{array}{l}\text { gauss norm } \\ \text { proper subset of } \\ \text { special set }\end{array} & 1.3 \\ \bar{K} & 6.1 & \text { special set } & 1.13 \\ \mathfrak{M} & 2.2 & \text { standard set } & 1.9 \\ M(A) & 1.5 & \text { super admissible } & 1.10,1.11 \\ \bigcirc_{E} & 6.2 & \text { very standard } & 1.9 \\ \Re & 1.4 & & \\ \Re_{A} & 1.5 & & \\ \Re_{0} & 1.4 & & \end{array}$

\section{Notation.}

1.1 Let $K$ be a field of characteristic zero complete under a nonarchimedean valuation and with residue class field of characteristic $p \neq 0$. Let $E_{0}=K(x)$ be the field of rational functions in one variable. Let $\Omega$ be an algebraically closed maximally complete field complete under a valuation extending that of $K$ and linearly disjoint from $E_{0}$ over $K$. Let $\Omega$ have a valuation ring containing an element $t$ whose image in the residue class field of $\Omega$ is transcendental over the residue class field of $K$. The point $t$ will be called the generic point.

1.2 For each $a \in \Omega$ and each positive real number $r$, let

$$
D\left(a, r^{-}\right)=\{x \in \Omega|| x-a \mid<r\}, \quad D\left(a, r^{+}\right)=\{x \in \Omega|| x-a \mid \leqslant r\} .
$$

For $f \in \Omega[[x-a]], f=\sum_{\nu=0}^{\infty} b_{\nu}(x-a)^{\nu}$, analytic on $D\left(a, r^{-}\right)$, let for $\rho<r$

$$
|f|_{a}(\rho)=\sup _{\nu}\left|b_{\nu}\right| \rho^{\nu}
$$

This is extended to functions $f$, meromorphic in $D\left(0, r^{-}\right)$by writing

$$
|f|_{a}(\rho)=|g|_{a}(\rho) /|h|_{a}(\rho) \text { if } f=g / h
$$

both $g$ and $h$ being analytic on $D\left(a, r^{-}\right)$.

1.3 Let $E$ be the completion of $E_{0}$ under the Gauss norm

$$
f \rightarrow|f|_{0}(1) .
$$

We shall write $|f|_{E}$ for this norm on $E$. The operator norm on the space of continuous endomorphisms of $E$ is also denoted by ||$_{E}$.

1.4 Let $\Re=E[D]$ be the noncommutative euclidean ring of finite sums, $(D=d / d x), \sum c_{m} D^{m}, c_{m} \in E$. Let $\Re_{0}$ be the subring $E_{0}[D]$.

1.5 For each bounded subset $A$ of $\Omega$ such that $d(A, \mathbf{C} A)>0$ (or the union of such a bounded set with the complement in $\Omega \cup\{\infty\}$ of $\mathbf{C} D(0, r)$ for some $r>0$ ). Let $G(A)$ be the subspace of $E_{0}$ consisting of rational functions having no poles in $A$ and let $H(A)$ denote the completion of $G(A)$ under the topology of uniform convergence. Let $M(A)$ be the quotient field of $H(A)$ and let 


$$
\Re_{A}=M(A)[D], \quad \mathfrak{S}_{A}=H(A)[D] .
$$

$H(A)$ is a banach space under the sup norm, \|\|$_{A}$, and the operator norm on the space of continuous endomorphism of $H(A)$ is denoted by the same symbol.

We define $H_{0}(A)$ to be the same as $H(A)$ unless $A$ contains the point at infinity in which case we impose the further condition of vanishing at infinity.

1.6 Let $\pi=\left(\pi_{\nu}\right)_{\nu \in N}$ be a nonincreasing sequence of positive real numbers with

$$
\pi_{0}=1, \quad \pi_{\nu} / \pi_{\nu+1} \text { monotonically decreasing. }
$$

Let $W_{a}^{\pi}$ be the banach space of germs of analytic functions at $a$,

$$
u=\sum_{\nu=0}^{\infty} b_{\nu}(x-a)^{\nu}
$$

such that

$$
\|u\|_{\pi}=\sup _{\nu} \pi_{\nu}\left|b_{\nu}\right|<+\infty
$$

with norm $u \mapsto\|u\|_{\pi}$.

1.7 We shall use $\mathbb{Q}_{a}^{\rho}$ to denote the space of functions analytic in $D\left(a, \rho^{-}\right)$ with topology of uniform convergence on disks $D\left(a, r^{-}\right)$with $r<\rho$. We write $\mathbb{Q}_{a}$ instead of $\mathbb{Q}_{a}^{1}$.

If $\Delta$ is an annulus of center $a$,

$$
\Delta=D\left(a, \rho_{2}^{-}\right)-D\left(a, \rho_{1}^{-}\right)
$$

where $\rho_{1}<\rho_{2}$ then let $\mathbb{Q}_{\Delta}$ denote the space of functions analytic on $\Delta$ with the topology of uniform convergence on annuli

$$
\Delta_{r}=D\left(a, r^{-}\right)-D\left(a, \rho_{1}^{-}\right)
$$

where $r \in\left(\rho_{1}, \rho_{2}\right)$.

Similarly if $\Delta=D\left(a, \rho_{2}^{+}\right)-D\left(a, \rho_{1}^{+}\right)$then again $\mathbb{Q}_{\Delta}$ denotes the space of functions analytic on $\Delta$ with topology of uniform convergence on annuli

$$
\Delta_{r}=D\left(a, \rho_{2}^{+}\right)-D\left(a, r^{+}\right)
$$

where $r \in\left(\rho_{1}, \rho_{2}\right)$.

1.8 The ring $\Re$ may be identified with a subring of the ring of continuous endomorphisms of $W_{t}^{\pi}$. The induced norm of $\Re$ will be denoted $R \mapsto\|R\|_{\pi}$. In particular if $\pi_{\nu}=1$ for all $\nu \in N$, we denote $\pi$ by $\pi^{1,0}$ and we write \|\|$_{1,0}$ for the corresponding norm and write $W_{t}^{1,0}$ for $W_{t}^{\pi}$ and $W_{a}^{1,0}$ for $W_{a}^{\pi}$.

More generally $W_{a}^{r, \alpha}$ denotes $W_{a}^{\pi}$ where $\pi$ is the sequence whose general member is $\pi_{\nu}=r^{\nu} /(\nu+1)^{\alpha}$. 
1.9 A nonempty subset $A$ of $\Omega \cup\{\infty\}$ will be called very standard if it is a union of residue classes. The set $A$ will be said to be standard if it is the union of a very standard set with a finite collection of annuli which lie in $D\left(0,1^{+}\right)$ and have outer radius unity and no outer circumferences. Thus we may define $e_{A}$ to be the minimum of the inner radii of these annuli and if $A$ is very standard we set $e_{A}=1$. The definition of standard set and $e_{A}$ is extended in an obvious way so as to remove distinctions between finite and infinite residue classes: Let $\Delta$ be the annulus

$$
\Delta=\left\{x\left|e^{-1} \geqslant\right| x \mid>1\right\}
$$

If $A$ is either the empty set or a standard set then we shall say that $A^{\prime}=A \cup \Delta$ is also standard and we define $e_{A^{\prime}}$ to be $e$ if $A$ is empty and to be $\min \left(e_{A}, e\right)$ otherwise. Thus in all cases $e_{A} \leqslant 1$. If $A$ is standard then there exists a natural identification of $H(A)$ with $H\left(A \cup D\left(t, 1^{-}\right)\right)$and so we may view $H(A)$ as a subring of $E$. Furthermore we may view $D\left(t, 1^{-}\right)$as a subset of each standard set although it will be convenient not to insist upon this formulation.

1.10 A standard subset $B$ of a standard set $A$ will be said to be an admissible subset if the difference $A-B$ lies in the union of a finite number of residue classes. An admissible subset will be said to be superadmissible if the two sets have the same image in the residue class field, extended so as to include the infinite residue class.

$1.11 \mathrm{An}$ element of $E$ is said to be admissible (resp. superadmissible) if it lies in $H(A)$ for some admissible (resp. superadmissible) subset, $A$, of $\Omega$. We denote by $E_{\text {ad }}$ (resp., $E_{\text {sad }}$ ) the set of all admissible (resp. superadmissible) elements of $E$. We thus have a field theoretic inclusion

$$
E \supset E_{\text {ad }} \supset E_{\text {sad }} \supset E_{0} .
$$

1.12 A subset, $A$, of $\Omega \cup\{\infty\}$ will be said to be special if it is either an uncircumferenced disk $D\left(0, b^{-}\right)$or an uncircumferenced annulus $D\left(0, b^{-}\right)$ $-D\left(0, c^{+}\right)(b>c)$. We define $e_{A}$ in the first case to be $\operatorname{Min}\left(b, b^{-1}\right)$ and in the second case to be $\operatorname{Min}\left(b, b^{-1}, c, c^{-1}\right)$. This agrees with the previous notation if $A$ is also a standard set.

If $A$ is a special set then we shall use $W_{A}$ to denote the subspace of $\Omega[[X]]$ (resp. Laurent series with coefficients in $\Omega$ ) which converge and are bounded in $A$. We shall use $\mathscr{W}_{A}$ to denote the closure of $\Omega[x]$ (resp: $\left.\Omega\left[x, x^{-1}\right]\right)$ in $W_{A}$. The space $\mathscr{W}_{A}$ coincides with the space of power series (resp. Laurent series) which converge in the circumferenced disk (resp. annulus).

1.13 A subset $B$ of a special set $A$ will be said to be proper if it is a special set which is also a disk (resp. annulus) and if the radius of $B$ (resp. maximal 
radius, resp. minimal radius of $B$ ) is strictly less (resp. strictly less, resp. strictly greater) than that of $A$.

The space, $\mathbb{Q}_{A}$, of analytic functions on $A$, i.e. of power series in $\Omega[[X]]$ (resp. Laurent series) which converge in $A$ is endowed with the topology of uniform convergence on proper subsets. If $A=D\left(0, b^{-}\right)$then $\mathcal{Q}_{A}$ is precisely the same as $\mathbb{Q}_{0}^{b}$.

2. Comparison of bounded kernels of a differential operator at a generic point and at an arbitrary point. Let $\Delta$ be the annulus $D\left(0,1^{-}\right)-D\left(0, b^{-}\right)$where $b$ is a fixed real number in the interval $(0,1)$.

If $L$ is an element of $\Re$ then we may consider solutions of $L$ at $t$ but it need not be possible to discuss solutions at points in $\Delta$. For this reason in $[11, \S 3]$ comparisons of solution spaces near different points were made only for differential operators in $\Re_{0}$. This restriction is unnecessarily restrictive. Following the same methods we show that such comparisons may be made in the case of $L \in \Re_{\Delta^{*}}$. Clearly

$$
\Re_{0} \subset \Re_{\Delta} \subset \Re
$$

2.1 Lemma. Let $L \in \Re_{\Delta}$, let $\pi$ be a sequence $(\S 1.6)$ and let $R$ be the monic generator of the $\pi$-closure in $\Re$ of $\Re L$. Let $R$ be of degree $k$ and for each $n \in \mathbf{N}$ let $R_{n}$ be monic of degree $k$ in $\Re_{0}$ such that

$$
\left\|R-R_{n}\right\|_{\pi}<1 / n
$$

There exists $P_{n} \in \Re_{\Delta}$ such that

$$
R_{n} \equiv P_{n} \bmod \Re_{\Delta} L
$$

$$
\left\|P_{n}\right\|_{\pi}<1 / n
$$

In particular if $R=1$, then $R_{n}=1$ for all $n$.

Proof. See [11, §3.1].

2.2 The boundary seminorm. If $u$ is a meromorphic function on $\Delta$, i.e. the ratio of two Laurent series which converge in $\Delta$, let

$$
\|u\|_{\sigma}=\lim \sup _{r \uparrow 1}|u|_{0}(r)=\lim _{r \uparrow 1} \sup _{s \in(r, 1)}|u|_{0}(s) .
$$

If $L \in \Re_{\Delta}$ then $L$ acts on endomorphism on the space of functions meromorphic on $\Delta$.

Let $\mathfrak{M}$ be the space of meromorphic functions on $\Delta$ of the form $g / h$ where $g$ and $h$ are functions analytic and bounded on $\Delta$. Then \|\|$_{\sigma}$ is a norm on $\mathfrak{D}$ and in particular for each $u \in \mathfrak{M}$ we have 


$$
\|u\|_{\sigma}<+\infty, \quad\|u\|_{\sigma}=0 \Leftrightarrow u=0 .
$$

We define the bounded meromorphic kernel of $L$ on $\Delta$, written, bdd mer $\operatorname{ker}_{\Delta} L$, to be the space of elements of $\mathfrak{M}$ annihilated by $L$.

2.3 Lemma. Let $L$ be an element of $\Re_{\Delta}$. If $u$ is meromorphic on $\Delta$, then

$$
\left\|u^{-1} L u\right\|_{\sigma} \leqslant\|L\|_{1,0} .
$$

If $u \in \mathfrak{M}$ then

$$
\|L u\|_{\sigma} \leqslant\|L\|_{1,0}\|u\|_{\sigma}
$$

Proof. See [11, §3.3].

2.4 We define the bounded kernel of $L$ at $t$ to be the kernel of $L$ in $W_{t}^{1,0}$, i.e. the space of those solutions of $L u=0$ such that $u$ is bounded on $D\left(t, 1^{-}\right)$.

THeOREM 2.4. (i) Let $L$ be a monic element of $\Re_{\Delta}$. Then

$$
\text { dim bdd } \operatorname{Ker}_{t} L \geqslant \operatorname{dim} \text { bdd } \operatorname{mer} \operatorname{Ker}_{\Delta} L .
$$

(ii) Let $M$ be the monic element of $\Re$ defined by

$$
\operatorname{Ker}_{t} M=\text { bdd } \operatorname{Ker}_{t} L \text {. }
$$

If equality holds in (2.4.1), then the coefficients of $M$ are analytic functions on $\Delta^{\prime} \cup D\left(t, 1^{-}\right)$where $\Delta^{\prime}$ is the complement in $\Delta$ of a countable set.

(iii) Let $A=D\left(0,1^{-}\right)$. If either $M \in \widetilde{S}_{A}$ or both $M \in \Re_{A}$ and $L \in \widetilde{S}_{A}$, then equality holds in (2.4.1).

Proof. For the proof of (i) see [11, §3.4]. For the proof of (iii) one simply follows the proof of [11, Lemma 4.25] replacing $k$ by 1 in equation (4.25.5) of that article. In the proof of (ii) we will use Proposition 2.6 whose statement and proof will be given at the end of this section. Let $k$ be the order of $M$. By hypothesis this is the same as the dimension of the bounded meromorphic kernel of $L$ on $\Delta$. Let $u_{1}, \ldots, u_{k}$ be a basis of this kernel. Note that $M$ does not a priori operate on $u_{i}$. We may normalize so that

$$
\left\|u_{i}\right\|_{\sigma}=1, \quad i=1,2, \ldots, k .
$$

Let $N$ be the monic differential operator, $N \in \mathfrak{M}[D]$, of order $k$, such that

$$
N u_{i}=0, \quad i=1,2, \ldots, k .
$$

By Lemma 2.1, for each $n \in \mathbf{N}$ there exists $M_{n} \in \Re_{0}, P_{n} \in \Re_{\Delta}$ such that $M_{n}$ is monic of degree $k$ and such that 


$$
\begin{aligned}
M_{n} & \equiv P_{n} \quad \bmod \Re_{\Delta} L, \\
\left\|M_{n}-M\right\|_{1,0} & \leqslant 1 / n, \\
\left\|P_{n}\right\|_{1,0} & \leqslant 1 / n .
\end{aligned}
$$

For $n \in \mathbf{N}, i=1,2, \ldots, k$, define

$$
M_{n} u_{i}=\theta_{n, i} .
$$

By equation (2.4.4.1), $M_{n} u_{i}=P_{n} u_{i}$ while by (2.4.4.3),

$$
\left\|P_{n} u_{i}\right\|_{\sigma} \leqslant 1 / n
$$

and thus

$$
\left\|\theta_{n, i}\right\|_{\sigma} \leqslant 1 / n .
$$

From equations (2.4.3), (2.4.5) we have

$$
\left(M_{n}-N\right) u_{i}=\theta_{n, i}, \quad i=1,2, \ldots, k .
$$

Now $M_{n}-N$ is a differential operator of order not greater than $k-1$, with coefficients in $\mathfrak{M}$. Solving (2.4.7) for these $k$ coefficients, and using (2.4.6) we find a constant $c$, independent of $n$ such that

$$
\left\|M_{n}-N\right\|_{\sigma} \leqslant c / n,
$$

where the symbol on the left is the maximal boundary norm of the coefficients of the indicated differential operator (i.e. if $\sum b_{i} D^{i}=\phi \in \mathfrak{M}[D]$ then $\|\phi\|_{\sigma}$ is by definition $\left.\operatorname{Max}_{i}\left\|a_{i}\right\|_{\sigma}\right)$. Now, $M, N, M_{n}$ being all monic of the same order, equations (2.4.8) and (2.4.4.2) together with Proposition 2.6 below show that the coefficients of $M$ extend to $\Delta$ as meromorphic functions and coincide on $\Delta$ with the coefficients of $N$. This completes the proof of the theorem.

2.5 TheOREM. Let $L \in \Re_{\Delta}$. If there exists $u \neq 0$ and meromorphic in $\Delta$ such that $L u=0$, then the bounded kernel of $L$ at $t$ is nontrivial.

Proof. See [11, §3.5].

2.6 To complete the proof of Theorem 2.4 above, we need the proof of the following proposition. The annulus $\Delta$, the set $\mathfrak{M}$ and the boundary norm $\sigma$ are defined in $\$ 2.2$.

Clearly $\mathfrak{M}$ contains $E_{0}$ and the boundary seminorm (2.2.1) induces a norm on $\mathfrak{M}$ which in turn induces the gauss norm on $E_{0}$. In this section we shall consider an element $f$ of $\mathfrak{M}$ which is the limit of elements of $E_{0}$. In this situation there is a unique element, $F$, of $E$ which is the limit in $E$ of the elements in $E_{0}$ which have $f$ as limit in $\mathfrak{M}$. 
Our object is to determine a sense in which $f$ is the "analytic continuation" of $F$. For this purpose, we need some new symbols. Let $f=g / h$ where $g$ and $h$ are bounded functions analytic on $\Delta$. For each $c \in(0,1)$, let

$$
\begin{aligned}
Z_{c} & =\left\{\left.x \in \Delta|| h(x)|\geqslant c| h\right|_{0}(|x|)\right\}, \\
Y_{c} & =Z_{c} \cup D\left(t, 1^{-}\right), \\
Y & =\bigcup_{c>0} Y_{c}, \\
A & =D\left(0,1^{-}\right), \\
D_{c} & =Z_{c} \cup \mathbf{C} A .
\end{aligned}
$$

It has been shown by Motzkin [8] that $Y_{c}$ is an analytic set and hence the same holds for $Y$.

Proposition 2.6. Let $g, h$ be bounded elements cf $\mathbb{Q}_{\Delta}$ whose quotient $f=g / h$ lies in the closure of $E_{0}$ in $\mathfrak{M}$. Then $f \in H\left(Y_{c}\right)$ for each $c \in(0,1)$. The continuation of $f$ to $D\left(t, 1^{-}\right)$is given by $F$ and $f$ is an analytic function on $Y$.

Proof. The group $G$ of continuous automorphisms of $\Omega$ over $K$ is stable on $\Delta$ and hence ( $\$ 8)$ acts as group of automorphisms of $\mathfrak{M}$. In this sense, the action of $G$ on $E_{0}$ is trivial and hence $f$ is invariant under $G$.

Let $(g),(h)$ denote the divisors of $g$ and $h$ as functions on $\Delta$ in the sense of Lazard (cf. Van der Put [15, Theorem 4.3]). Since $\Omega$ is maximally complete, there exists $\xi \in W_{A}$, such that $(\xi)$ is the greatest common divisor of $(g)$ and $(h)$. We may replace $g$ (resp. $h$ ) by $g / \xi($ resp. $h / \xi$ ) and may assume that $g$ and $h$ are bounded analytic functions on $\Delta$ with no common zeros. There is no loss in generality as $Z_{c}$ is replaced by a larger set.

We know that $f=g / h$ is invariant under $G$ but do not know whether the same holds for $h$. However it is now clear that $\left(h^{\tau}\right)=(h)$ for each $\tau \in G$ and hence

$$
h^{\tau}=h \cdot \eta_{\tau}
$$

where $\eta_{\tau}$ is an element of $W_{\Delta}$ which has no zeros in $\Delta$. Thus, for $x \in \Delta$ we have

$$
\left|\eta_{\tau}(x)\right|=\left|\eta_{\tau}\right|_{0}(|x|)
$$

But $\tau$ induces a permutation of $\{x|| x \mid=r\}$ and hence

$$
\left|h^{\tau}\right|_{0}(r)=|h|_{0}(r)
$$

for each $r \in[b, 1)$. From these relations, we deduce that $\left|\eta_{\tau}\right|_{0}(r)=1$ and that $\left|\eta_{\tau}(x)\right|=1$ for all $x \in \Delta$. This shows that the sets $Z_{c}, Y_{c}, D_{c}$ are all stable under $G$. 
The set $Z_{c}$ has $D\left(0, b^{-}\right)$as a trou and there exists $f_{0}$ analytic element on C $D\left(0, b^{-}\right)$vanishing at $\infty$ such that $f-f_{0}$ has analytic continuation to $D\left(0, b^{-}\right)$. Since $f_{0}$ is uniquely specified by these properties, it is invariant under $G$ and using the representation of $f_{0}$ as element of $\Omega((1 / x))$ it follows from Theorem 8 that $f_{0} \in H_{0}\left(\mathbf{C} D\left(0, b^{-}\right)\right)$. The other trous of $Z_{c}$ lie on circumferences

$$
\Gamma_{n}=\left\{x|| x \mid=r_{n}\right\}
$$

where $h$ has zeros. The sequence $\left\{r_{n}\right\}$ if infinite is monotonic with 1 as limit. For each zero $\alpha$ of $h$ let $\xi_{\alpha}(\in \Omega(x))$ be the singular part of $f$ at $\alpha$. For each $n \geqslant 1$, we define

$$
f_{n}=\sum \xi_{\alpha}
$$

the sum being over all $\alpha \in \Gamma_{n}$ such that $h(\alpha)=0$. Clearly $f_{n}$ is an element of $\Omega(x)$ which is invariant under $G$ and hence $f_{n} \in K(x)$. Clearly $f_{n} \in H_{0}\left(D_{c}\right)$ for $n \geqslant 0$.

Our first object is to prove

$$
\left\|f_{n}\right\|_{D_{c}} \rightarrow 0 .
$$

Given $\varepsilon>0$, there exists $Q \in E_{0}$ such that

$$
|F-Q|_{E}<c \varepsilon, \quad\|f-Q\|_{\sigma}<c \varepsilon .
$$

We may choose $r \in(b, 1)$ such that $r \neq r_{m}$ for any $m$ and such that

$$
|f-Q|_{0}(\rho)<c \varepsilon \quad \forall \rho \in(r, 1),
$$

$$
Q \text { has no pole } \beta \text { such that }|\beta| \in[r, 1) \text {. }
$$

Let $r_{n} \in(r, 1)$. Choose $r_{n}^{\prime}, r_{n}^{\prime \prime} \in(0,1)$ such that

$$
\operatorname{Max}\left(r, r_{n-1}\right)<r_{n}^{\prime}<r_{n}<r_{n}^{\prime \prime}<r_{n+1} .
$$

Thus $Q$ has no poles in the annulus

$$
\left\{x\left|r_{n}^{\prime}<\right| x \mid<r_{n}^{\prime \prime}\right\}
$$

and the poles of $f$ in this annulus lie on $\Gamma_{n}$. Let $V$ be the intersection of this annulus with $Z_{c}$. Corresponding to each trou $T$ of $V$ on $\Gamma_{n}$ the singular part of $f-Q$ is

$$
(f-Q)_{T}=f_{T}=\sum_{\alpha \in T} \xi_{\alpha},
$$

From the Mittag-Leffler theorem [6], 


$$
\left\|(f-Q)_{T}\right\|_{\mathbf{C} T} \leqslant\|f-Q\|_{V} .
$$

To estimate the right-hand side of (2.6.7), we consider two cases

$1^{\circ}$. For $x \in V, x \notin \Gamma_{n}$, by (2.6.5), we have

$$
|(f-Q)(x)| \leqslant|f-Q|_{0}(|x|)<c \varepsilon<\varepsilon .
$$

$2^{\circ}$. For $x \in V \cap \Gamma_{n}$, by definition of $Z_{c}$

$$
|h(x)| \geqslant c|h|_{0}\left(r_{n}\right)
$$

while the analyticity of $g-h Q$ on $\Gamma_{n}$ shows that

$$
|(g-h Q)(x)| \leqslant|g-h Q|_{0}\left(r_{n}\right) .
$$

Thus again by (2.6.5),

$$
|(f-Q)(x)| \leqslant|f-Q|_{0}\left(r_{n}\right) / c<\varepsilon .
$$

This shows that

$$
\|f-Q\|_{V}<\varepsilon .
$$

By (2.6.6), (2.6.7), (2.6.8), we see that

$$
\left\|f_{T}\right\|_{C T}<\varepsilon .
$$

Since $\mathbf{C} T \supset D_{c}$, we conclude that

$$
\left\|f_{T}\right\|_{D_{c}}<\varepsilon .
$$

The trous of $V$ on $\Gamma_{n}$ contain all the zeros of $h$ on $\Gamma_{n}$ and thus by (2.6.2), (2.6.6),

$$
f_{n}=\sum f_{T}
$$

the sum on the right being over all trous $T$ of $V$ on $\Gamma_{n}$. It now follows from (2.6.10) that

$$
\left\|f_{n}\right\|_{D_{c}}<\varepsilon \quad \forall r_{n} \in(r, 1)
$$

This completes the proof of (2.6.3).

We now define

$$
f_{A}=\sum_{n=0}^{\infty} f_{n},
$$

an element of $H\left(D_{c}\right)$. Let $F_{A}$ be the singular part of $F$ corresponding to the trou, $A$, of $D\left(t, 1^{-}\right)$. Thus 


$$
F_{A} \in H(\mathbf{C} A), \quad F-F_{A} \in H\left(A \cup D\left(t, 1^{-}\right)\right) .
$$

Our second object is to show that

$$
F_{A}=f_{A}
$$

as elements of $H(\mathbf{C} A)$.

Let $Q$ be an element of $E_{0}$ and $r \in(b, 1)$ as chosen above. Let $Q_{A}$ be the singular part of $Q$ corresponding to poles of $Q$ in $A$. Viewing $A$ as a trou of $D\left(t, 1^{-}\right)$, we conclude from (2.6.4) and the Mittag-Leffler theorem that

$$
\left\|F_{A}-Q_{A}\right\|_{C_{A}} \leqslant\|F-Q\|_{D\left(t, 1^{-}\right)}<\varepsilon .
$$

Now, consider the restriction of $f$ and of $Q$ to the circumference $\Gamma$ $=\{x|| x \mid=r\}$. Since all the poles of $Q$ in $A$ lie in $D\left(0, r^{-}\right)$, the singular part of $(f-Q) \mid \Gamma$ relative to the trou $D\left(0, r^{-}\right)$of $\Gamma$ is

$$
(f-Q)_{D\left(0, r^{-}\right)}=\sum_{r_{m}<r} f_{m}-Q_{A}
$$

it being understood that $f_{0}$ is the first term in the sum on the right. By the Mittag-Leffler theorem and equation (2.6.5),

$$
\left\|(f-Q)_{D\left(0, r^{-}\right)}\right\|_{C D\left(0, r^{-}\right)} \leqslant\|f-Q\|_{\Gamma}=|f-Q|_{0}(r)<\varepsilon .
$$

Since $\mathbf{C} D\left(0, r^{-}\right) \supset \mathbf{C} A$, the last two equations give

$$
\left\|\sum_{r_{m}<r} f_{m}-Q_{A}\right\|_{\mathbf{C}_{A}}<\varepsilon,
$$

and since $D_{c} \supset \mathrm{C} A$, it follows from (2.6.12) that

$$
\left\|f_{n}\right\|_{C_{A}}<\varepsilon \quad \forall r_{n} \geqslant r .
$$

We conclude that

$$
\left\|f_{A}-Q_{A}\right\|_{C_{A}}<\varepsilon .
$$

Equation (2.6.15) now follows from (2.6.16) and (2.6.21), which completes the proof of our second object.

We note that $f-f_{A}$ is an analytic function on $Z_{c}$ which has a unique extension to a bounded analytic function on $A$. As noted in (2.6.14), $F-F_{A}$ has continuation into $A$. Our third object is to show that

$$
F-F_{A}=f-f_{A}
$$

as functions on $A$. With $Q$ as chosen above, we use 


$$
\begin{aligned}
\left\|\left(f-f_{A}\right)-\left(Q-Q_{A}\right)\right\|_{A} & =\left\|\left(f-f_{A}\right)-\left(Q-Q_{A}\right)\right\|_{\sigma} \\
\left\|f_{A}-Q_{A}\right\|_{\sigma} & =\left\|f_{A}-Q_{A}\right\|_{C_{A}},
\end{aligned}
$$

and equations (2.6.4) and (2.6.21) to conclude that

$$
\left\|\left(f-f_{A}\right)-\left(Q-Q_{A}\right)\right\|_{A}<\varepsilon
$$

we also use

$$
\left\|\left(F-F_{A}\right)-\left(Q-Q_{A}\right)\right\|_{A}=\left\|\left(F-F_{A}\right)-\left(Q-Q_{A}\right)\right\|_{E}
$$

and equations (2.6.4) and (2.6.16) to conclude that

$$
\left\|\left(F-F_{A}\right)-\left(Q-Q_{A}\right)\right\|_{A}<\varepsilon .
$$

Equation 2.6.22 is a consequence of (2.6.23) and (2.6.24). For $x \in Z_{c}$, we have by $(2.6 .22)$

$$
f(x)=f_{A}(x)+\left(F-F_{A}\right)(x) .
$$

Since $f_{A} \in H\left(D_{c}\right)$ and $F-F_{A} \in H\left(A \cup D\left(t, 1^{-}\right)\right)$, it follows that $f$ is the restriction to $Z_{c}$ of an element of $H\left(Y_{c}\right)$ and this element is unique since $Y_{c}$ is an analytic set. Denoting this extended function again by $f$, equation $(2.6 .25)$ extends to $Y_{c}$ and in particular is valid for $x \in D\left(t, 1^{-}\right)$. But for such $x$, we have by $(2.6 .15) f_{A}(x)=F_{A}(x)$ and hence $f(x)=F(x)$.

This shows that the extension of $f$ to $D\left(t, 1^{-}\right)$is given by $F$ as asserted. This completes the proof of the theorem.

3. Nonlinear differential equations with a solution in $E$. The object of this section is the study of nonlinear differential equations having coefficients which are analytic elements on a standard set $A$.

3.1 Let $s, m$ be fixed positive integers (in the applications $s=m$ ) and let $\rho$ be a fixed positive real number. Let $F$ be an analytic map of $A \times\left(D\left(0, \rho^{+}\right)\right)^{2 s}$ into $\Omega^{m}$ defined over $K$, i.e. letting $X=\left(X_{1}, \ldots, X_{s}\right), Y=\left(Y_{1}, \ldots, Y_{s}\right)$ then

$$
F(X, Y)=\sum_{\mu, \nu} C_{\mu, \nu} X^{\mu} Y^{\nu}
$$

the sum being over all $\mu$ and $\nu$ in $\mathbf{Z}_{+}^{s}$, it being understood that for $\mu \in \mathbf{Z}_{+}^{s}, X^{\mu}$ represents the monomial $X_{1}^{\mu_{1}} \cdots X_{s}^{\mu_{s}}$. Furthermore each $C_{\mu, \nu} \in(H(A))^{m}$, i.e. is an $m$-vector whose components lie in $H(A)$ and which are all functions of the same independent variable $x$. It is assumed that the series converges whenever $x \in A$ and each $X_{i}$ and each $Y_{i}$ lies in $D\left(0, \rho^{+}\right)$. In particular then there exists a real number $M$ such that

$$
\rho^{|(\mu, \nu)|}\left\|C_{\mu, \nu}\right\|_{A} \leqslant M
$$


for all $(\mu, \nu) \in \mathbf{Z}_{+}^{2 s}$ where $|(\mu, \nu)|=\sum_{i=1}^{s} \mu_{i}+\sum_{i=1}^{s} \nu_{i}$ and $\left\|C_{\mu, \nu}\right\|_{A}$ denotes the maximum of the norms of the components of $C_{\mu, \nu}$ as elements of $H(A)$.

Let $u=\left(u_{1}, \ldots, u_{s}\right)$ be a solution in $E^{s}$ of the system

$$
F(X, d X / d x)=0
$$

such that $|u|_{E}=\sup _{1<i<s}\left|u_{i}\right|_{E}<\rho$.

We define the tangential map of $F$ at $u$ to be the map of $E^{s}$ into $E^{m}$ given explicitly by

$$
L_{u}\left(z_{1}, \ldots, z_{s}\right)=\sum_{i=1}^{s} z_{i} \frac{\partial F}{\partial X_{i}}\left(u, u^{\prime}\right)+\sum_{i=1}^{s} z_{i}^{\prime} \frac{\partial F}{\partial Y_{i}}\left(u, u^{\prime}\right)
$$

where $z_{i}^{\prime}=d z_{i} / d x, u^{\prime}=d u / d x$.

3.1.5 CONJECTURE. If the mapping $z \mapsto L_{u}(z)$ has kernel of finite dimension (over $\Omega$ ) in the space of $s$-tuples of germs of analytic functions at $t$ then $u \in H(B)^{s}$ for some admissible subset $B$ of $A$.

This conjecture is known only in very special cases (cf. (3.7), (9.2) below).

The object of this section is to prove a stronger conclusion starting from a stronger premise.

3.1.6 TheOREM. If $L_{u}$ is injective on $\left(\mathbb{Q}_{t}\right)^{s}$, then $u \in H(B)^{s}$ for some superadmissible subset $B$ of $A$.

The proof will be completed in $\$ 3.6$ below.

3.2 Generalities concerning power series. Let $A, F$ be as in the preceding paragraph. Let $B$ be a standard subset of $A$. For $X \in H(B)^{s}$, let $G(X)$ $=F(X, d X / d x)$. If now $\|X\|_{B}<\rho e_{B}$ then $\|d X / d x\|_{B}<\rho$ and we may consider the Taylor expansion of $G$ about $X$ in the banach space $(H(B))^{s}$ and hence we may write for $\xi \in H(B)^{s}, \xi$ small,

$$
G(X+\xi)=G(X)+L_{X}(\xi)+N_{X}(\xi)
$$

where $L_{X}(\xi)$ (as given by (3.1.4)) gives the linear terms in $(\xi, d \xi / d x)$ and $N_{X}(\xi)$ give the sum of the nonlinear terms.

LEMMA. For $\xi, \tilde{\xi}$ in $H(B)^{s}$

$$
\operatorname{Max}\left(\|\xi\|_{B},\|\tilde{\xi}\|_{B}\right)<\rho e_{B}
$$

we have

$$
\begin{gathered}
\left\|L_{X}(\xi)\right\|_{B} \leqslant \frac{M}{\rho e_{B}}\|\xi\|_{B}, \quad\left\|N_{X}(\xi)\right\|_{B} \leqslant \frac{M}{\left(\rho e_{B}\right)^{2}}\|\xi\|_{B}^{2} \\
\left\|N_{X}(\xi)-N_{X}(\tilde{\xi})\right\|_{B} \leqslant \frac{M}{\left(\rho e_{B}\right)^{2}}\|\xi-\tilde{\xi}\|_{B} \sup \left(\|\xi\|_{B},\|\tilde{\xi}\|_{B}\right) .
\end{gathered}
$$


Proof. Similar inequalities for $F(X, Y)$ are well known consequences of the Cauchy inequality. The present relations are now a consequence of the estimate

$$
\|d \xi / d x\| \leqslant\|\xi\|_{B} / e_{B}
$$

3.3 Lemma. Let $C$ be a standard set, let $L \in \mathbb{S}_{C}$ (i.e. $L$ is a linear differential operator with coefficients in $H(C))$. For each real $\varepsilon>0$ there exists $e \in(0,1)$ such that for each standard subset $B$ of $C$ for which $e_{B}>e$ we have

$$
\|L\|_{B} \leqslant\|L\|_{1,0}+\varepsilon \text {. }
$$

Proof. $L=\sum c_{m} D^{m} / m !$, let $d=d(B, \mathbf{C} B)$, then

$$
\|L\|_{B} \leqslant \operatorname{Max}_{m} d^{-m}\left\|c_{m}\right\|_{B} \leqslant \operatorname{Max}_{m} e_{B}^{-m}\left\|c_{m}\right\|_{B} .
$$

Let $\bar{\alpha}$ be an arbitrary residue class of $\Omega$ which intersects $C$. If $\bar{\alpha} \subset C$ then for each $x \in \bar{\alpha}$ we have

$$
\left|c_{m}(x)\right| \leqslant\left|c_{m}\right|_{E} .
$$

If on the contrary $\bar{\alpha} \llbracket C$ then $\bar{\alpha} \cap \mathbf{C} C$ is a disk. If $\bar{\alpha} \neq \infty$ (resp. $\bar{\alpha}=\infty$ ) let $\alpha$ be an element of this disk (resp. let $\alpha=0$ ). Since $\left|c_{m}\right|_{\alpha}(r)$ approaches $\left|c_{m}\right|_{E}$ as $r \uparrow 1$ (resp. $r \downarrow 1)$ there exists $\gamma_{\alpha} \in(0,1)$ such that

$$
\left|c_{m}\right|_{\alpha}(r) \leqslant\left|c_{m}\right|_{E}+\varepsilon / 2
$$

for all $m$ and all $r \in\left(\gamma_{\alpha}, 1\right)\left(\right.$ resp. $\left.\left(1, \gamma_{\alpha}^{-1}\right)\right)$. Since there are only a finite number of residue classes $\bar{\alpha}$ of this second type (i.e. such that $\bar{\alpha} \cap C \neq \varnothing, \bar{\alpha} \nsubseteq C$ ) we may conclude that there exists $e^{\prime} \in(0,1)$ such that if $B$ is a standard subset of $C$ and $e_{B} \in\left(e^{\prime}, 1\right)$ then

$$
\left|c_{m}(x)\right| \leqslant\left|c_{m}\right|_{E}+\varepsilon / 2
$$

for all $x \in B$. We now choose $e \in\left(e^{\prime}, 1\right)$ such that

$$
e^{-m}<1+\varepsilon\left(\left|c_{m}\right|_{E}+\varepsilon\right)^{-1} \cdot \frac{1}{2}
$$

for all $m$ such that $c_{m} \neq 0$. If $e_{B}>e$ then equation (3.3.1) follows from (3.3.2), (3.3.3) and this last inequality since

$$
\|L\|_{1,0}=\operatorname{Max}_{m}\left|c_{m}\right|_{E} .
$$

3.3.4 Corollary. Let $C$ be a standard set and let $L$ be an $n \times n$ matrix with 
coefficients in $\Im_{C}$. For each $\varepsilon>0$ there exists $e \in(0,1)$ such that if $B$ is a standard subset of $C$ with $e_{B}>e$ then

$$
\|L\|_{B} \leqslant\|L\|_{1,0}+\varepsilon .
$$

3.4 Lemma. Let $L$ be an $n \times n$ matrix with coefficients in $\Re$. Let $L$ be injective as endomorphism of $\left(\mathbb{Q}_{t}\right)^{n}$. Then there exists an $n \times n$ matrix $Q$ with coefficients in $\Re_{0}$ such that

$$
\|Q L-I\|_{1,0}<1 .
$$

Furthermore $Q$ is also injective on $\left(Q_{t}\right)^{n}$.

Proof. By the theory of elementary divisors there exist invertible $n \times n$ matrices $U, V$ with coefficients in $\Re$ such that

$$
U L V=J,
$$

where $J$ is a diagonal matrix. Let $\alpha_{i} \in \Re$ denote the $i$ th diagonal entry. As $L$ is injective in $\left(Q_{t}\right)^{n}$ the same must hold for $J$ and hence each $\alpha_{i}$ must be injective on $\mathscr{Q}_{t}$. Certainly $\alpha_{i}$ must be injective on $W_{t}^{1,0}$ and hence by $[11, \S 2.6]$ there exists $\beta_{i} \in \Re$ such that for each $i$,

$$
\left\|\beta_{i} \alpha_{i}-1\right\|_{1,0}<1 /\|V\|_{1,0}\left\|V^{-1}\right\|_{1,0} .
$$

Let $J^{\prime}$ be diagonal $n \times n$ matrix with $\beta_{i}$ as $i$ th diagonal entry, and put $Q^{\prime}=V J^{\prime} U$. Then

$$
Q^{\prime} L-I=V\left(J^{\prime} U L V-I\right) V^{-1}=V\left(J^{\prime} J-I\right) V^{-1}
$$

and $J^{\prime} J-I$ is diagonal with $\beta_{i} \alpha_{i}-1$ as $i$ th diagonal entry. Thus

$$
\left\|Q^{\prime} L-I\right\|_{1,0} \leqslant\|V\|_{1,0} \sup _{i}\left\|\beta_{i} \alpha_{i}-1\right\|_{1,0}\left\|V^{-1}\right\|_{1,0}<1 .
$$

We now choose $Q$ an $n \times n$ matrix with coefficients in $\Re_{0}$ such that

$$
\left\|Q^{\prime}-Q\right\|_{1,0}<1 /\|L\|_{1,0} .
$$

Equation (3.4.1) follows from (3.4.4) and (3.4.5). Thus $Q L$ is invertible on $\left(W_{t}^{1,0}\right)^{n}$ and hence $Q$ is surjective. Again $Q$ is equivalent to a diagonal matrix, i.e. $Q=\Phi \Gamma \Theta$ where $\Phi$ and $\Theta$ are invertible $n \times n$ matrices with coefficients in $\Re$ and $\Gamma$ is diagonal. Thus $\Gamma$ is also surjective on $\left(W_{t}^{1.0}\right)^{n}$ and hence by [11, 4.10] each of the diagonal coefficients of $\Gamma$ is injective on $W_{t}^{1,0}$. Thus $\Gamma$ and therefore $Q$ are also injective on $\left(W_{t}^{1,0}\right)^{n}$. This completes the proof of the Lemma. 
3.4.6 Proposition. Let $V$ be a topological vector space, let $\theta$ and $\phi$ be endomorphisms of $V$ such that

(i) $\theta$ is injective.

(ii) $\theta \phi$ is automorphism of $V$.

Then $\phi($ and $\theta)$ are automorphisms of $V$.

Proof. By (ii) $\theta$ is surjective and hence by (i), $\theta$ is an automorphism of the algebraic structure of $V$. But $I=\theta \phi(\theta \phi)^{-1}$ shows that the continuous mapping, $\phi(\theta \phi)^{-1}$, is the inverse of $\theta$. This completes the proof.

3.5 We now return to the notation of Theorem 3.1.6. Since $L_{u}$ is an injection of $\left(\mathbb{Q}_{t}\right)^{s}$ into $\left(\mathbb{Q}_{t}\right)^{m}$ it follows from the theory of elementary divisors that $m \geqslant s$. If $m>s$ then by discarding suitably chosen $m-s$ components of $F$ we may reduce to the case in which $s=m$. Thus we may assume that $m=s$.

Since $L_{u}$ is injective on $\left(Q_{t}\right)^{m}$ we know from Lemma 3.4 that there exists $Q$, an $m \times m$ matrix with coefficients in $\Re_{0}$ such that

$$
\left\|Q L_{u}-I\right\|_{1,0}<1
$$

We choose real numbers $\sigma, q, w$ such that

$$
\begin{aligned}
\sigma & >\|Q\|_{1,0}, \\
q & <\rho \operatorname{Min}(1, \rho / \sigma M), \\
w & <q \operatorname{Min}(1, \rho / \sigma M) .
\end{aligned}
$$

(In fact $\rho /(\sigma M)<1$ since by (3.5.1), $1=\left\|Q L_{u}\right\|_{1,0}<\sigma\left\|L_{u}\right\|_{1,0}$ while by 3.2, $\left\|L_{u}\right\|_{1,0} \leqslant M / \rho$.) We choose $\eta \in E_{0}^{m}$ such that

$$
\begin{gathered}
|u-\eta|_{E}<w, \\
\left\|L_{\eta}-L_{u}\right\|_{1,0}<1 / \sigma .
\end{gathered}
$$

LEMma 3.5. There exists a superadmissible subset $B$ of $A$ such that $L_{\eta}$ is invertible as endomorphism of $H(B)^{m}$ and such that (with $G$ as defined in \$3.2)

$$
\begin{gathered}
\left\|L_{\eta}^{-1}\right\|_{B}<\sigma, \\
\|G(\eta)\|_{B}<w M / \rho,
\end{gathered}
$$

and such that

$$
\begin{aligned}
q & <\rho e_{B} \operatorname{Min}\left(1, \rho e_{B} / \sigma M\right), \quad\|u\|_{E}<\rho e_{B} \\
w & <q \operatorname{Min}\left(1, \rho e_{B} / \sigma M\right) .
\end{aligned}
$$


Proof. $Q L_{\eta}-I=\left(Q L_{u}-I\right)+Q\left(L_{\eta}-L_{u}\right)$ and hence by (3.5.1), (3.5.2) and (3.5.6) we have

$$
\left|Q L_{\eta}-I\right|_{E} \leqslant\left\|Q L_{\eta}-I\right\|_{1,0}<1 .
$$

As noted in Lemma 3.4, $Q$ is injective in $\left(W_{t}^{1,0}\right)^{m}$ and hence is injective as endomorphism of $E^{m}$. Equation (3.5.10) shows that $Q L_{\eta}$ is invertible on $E^{m}$ and hence by Proposition 3.4.6 $L_{\eta}$ is also invertible on $E^{m}$. Writing $Q L_{\eta}$ $=I+J$ then $|J|_{E}<1$ and hence

$$
\left|L_{\eta}^{-1}\right|_{E}=\left|(I+J)^{-1} Q\right|_{E} \leqslant|Q|_{E} \leqslant\|Q\|_{1,0}<\sigma .
$$

By hypothesis $|u|_{E}<\rho$ and so by (3.5.3), (3.5.4), (3.5.5), $|\eta|_{E}<\rho$. Applying Corollary 3.3.4 to $Q$, to $Q L_{\eta}-I$ and to $\eta$ we conclude that if $B$ is a superadmissible subset of $A$ containing no pole of either $\eta$ or of any of the coefficients of $Q$ and if $e_{B}$ is sufficiently close to unity then

$$
\begin{aligned}
\|Q\|_{B} & <\sigma, \\
\left\|Q L_{\eta}-I\right\|_{B} & <1, \\
\|\eta\|_{B} & <\rho e_{B} .
\end{aligned}
$$

Once again equation (3.5.13) shows that $Q L_{\eta}$ is invertible as endomorphism of $H(B)^{m}$. But $Q$ is also injective on this space as $H(B) \subset W_{t}^{1,0}$, and hence by Proposition 3.4.6 is invertible on $H(B)^{m}$. Furthermore with $J$ as in (3.5.11), we now know that $\|J\|_{B}<1$ and hence by (3.5.12),

$$
\left\|L_{\eta}^{-1}\right\|_{B}=\left\|(I+J)^{-1} Q\right\|_{B} \leqslant\|Q\|_{B}<\sigma,
$$

which proves equation (3.5.7). Equation (3.5.9) follows from (3.5.3), (3.5.4) if $e_{B}$ is sufficiently close to unity. Finally in the notation of $\$ 3.2$,

$$
0=G(\eta)+L_{\eta}(u-\eta)+N_{\eta}(u-\eta)
$$

and hence by Lemma 3.2

$$
|G(\eta)|_{E} \leqslant \sup \left(M|u-\eta| / \rho, M|u-\eta|^{2} / \rho^{2}\right) .
$$

Since

$$
|u-\eta|_{E}<w<q<\rho
$$

it is clear that

$$
\|G(\eta)\|_{1,0}=|G(\eta)|_{E}<M w / \rho .
$$


Equation (3.5.8) now follows from Corollary 3.3.4 provided $e_{B}$ is sufficiently close to unity. This completes the proof of the lemma.

Note. Equation (3.5.17) is based on the fact that the natural inclusion of $E$ in $W_{t}^{1,0}$ is an isometry. A corresponding result for differential operators will be proven in $\$ 5$ below.

3.6 Proof of Theorem 3.1.6. Let $\lambda=G(\eta)$. Let $B$ be the superadmissible subset of $A$ whose existence has been demonstrated in Lemma 3.5. Let

$$
U_{E}=\left\{\left.z \in E^{m}|| z\right|_{E} \leqslant q\right\}, \quad U_{B}=\left\{z \in H(B)^{m} \mid\|z\|_{B} \leqslant q\right\} .
$$

Clearly $U_{B} \subset U_{E}$ and since $q<\rho e_{B}$ we conclude that $N_{\eta}(z)$ takes values in $E^{m}$ (resp. $H(B)^{m}$ ) for $z \in U_{E}$ (resp. $U_{B}$ ). Thus the mapping

$$
\phi: z \mapsto-L_{\eta}^{-1}\left(\lambda+N_{\eta}(z)\right)
$$

is well defined on $U_{E}$ and on $U_{B}$.

It follows from (3.5.7), (3.5.8), (3.5.9) and Lemma 3.2 that

$$
\left\|L_{\eta}^{-1}(\lambda)\right\|_{B} \leqslant \sigma w M / \rho<q
$$

and that

$$
\left\|L_{\eta}^{-1} N_{\eta}(z)\right\|_{B} \leqslant \sigma M q^{2} / e_{B}^{2} \rho^{2}<q
$$

for all $z \in U_{B}$. This shows that $\phi$ is stable on $U_{B}$. Similar estimates based upon 3.5.11, 3.5.17, 3.5.9, Lemma 3.2 show that $\phi$ is also stable on $U_{E}$. Let $\theta=\sigma M q /\left(\rho e_{B}\right)^{2}$. By (3.5.9), $\theta<1$. If $z, \zeta$ are elements of $U_{B}$ then by Lemma 3.2

$$
\|\phi(z)-\phi(\zeta)\|_{B}=\left\|L_{\eta}^{-1}\left(N_{\eta}(\zeta)-N_{\eta}(z)\right)\right\|_{B} \leqslant \theta\|z-\zeta\|_{B} .
$$

Thus $\phi$ is contractive on $U_{B}$ and by the same argument $\phi$ is contractive on $U_{E}$. Thus $\phi$ has a unique fixed point, $z_{B}$, in $U_{B}$ and a unique fixed point, $z_{E}$ in $U_{E}$. As $U_{B} \subset U_{E}$ the uniqueness implies that $z_{E}=z_{B}$. Now equation (3.5.15) shows that $u-\eta$ is a fixed point of $\phi$ which by (3.5.16) lies in $U_{E}$. This shows that $u-\eta=z_{E}=z_{B}$ lies in $U_{B}$ and hence in $H(B)^{m}$. Since $\eta \in E_{0}^{m}$ and $B$ contains no pole of $\eta$, it follows that $\eta$ lies in $H(B)^{m}$ and thus $u$ lies in $H(B)^{m}$. This completes the proof of the theorem.

3.6.5 Note. If $\bar{\alpha}$ is a residue class contained by $A$ then $\bar{\alpha}$ is contained by $B$ unless $\bar{\alpha}$ contains a pole of either $\eta$ or of one of the coefficients of $Q$.

3.7 We may deduce the verification of a special case of Conjecture 3.1.5. Suppose in (3.1.1), $F(X, Y)$ is independent of $Y$. Then $u$ in (3.1.3) is a solution of a finite set of analytic equations. In this case the hypotheses of Conjecture 
3.1.5 and Theorem 3.1.6 coincide and thus the conjecture in this case may be deduced from the theorem.

4. Comparison of radii of convergence of solutions of a linear differential operator at the generic point and at an arbitrary point. We now generalize results of an earlier article [3] which were available only for elements of $\Re_{0}$.

4.1 Let $A$ be a standard set. Let $L \in \Re_{A}$ and let $r$ be a real number, $r \in(0,1]$. Let $\operatorname{ker}_{t} L$ denote the kernel of $L$ operating on germs of analytic functions at $t$. The intersection of $\operatorname{ker}_{t} L$ with $\mathbb{Q}_{t}^{r}$ determines a monic factor $M$ of $L$ and by Theorem 2.6 [11] we have

$$
\begin{aligned}
L & =N \cdot M, \\
\operatorname{ker}_{t} M & =\mathbb{Q}_{t}^{r} \cap \operatorname{ker}_{t} L
\end{aligned}
$$

$M$ and $N$ lie in $\Re$.

THEOREM. There exists a superadmissible subset $B$ of $A$ such that $M \in \Re_{B}$.

Proof. Equation (4.1.1) is equivalent to the assertion that the coefficients of $M$ and $N$ satisfy a system of nonlinear differential equations. Let $m$ be the order of $M$ and $n$ the order of $N$. We may assume that $M$ and $N$ are both monic. The tangential map is

$$
(P, Q) \mapsto N \cdot Q+P \cdot M
$$

where $P$ (resp. $Q$ ) denotes a linear differential operator of order not greater than $n-1$ (resp. $m-1$ ). We assert that this mapping is an injective on $\left(Q_{t}[D]\right)_{n-1} \times\left(Q_{t}[D]\right)_{m-1}$, the subscripts indicating the bounds on the orders. Suppose otherwise. Then there exist $Q, P \neq 0$ in $\mathbb{Q}_{t}[D]$ with degrees bounded as indicated such that

$$
N Q=-P M .
$$

Now the right side annihilates $\operatorname{ker}_{t} M$, a space of dimension $m$. Hence $N Q$ annihilates this space. But $Q$ is of degree strictly less than $m$ and hence cannot annihilate this space. Thus there exists a nonzero germ $v$ at $t$ such that

$$
\begin{aligned}
N v & =0, \\
v & =Q w, \\
w & \in \operatorname{ker}_{t} M .
\end{aligned}
$$

By (4.1.7) $w \in \mathbb{Q}_{t}^{r}$ and since the coefficients of $Q$ lie in $\mathbb{Q}_{t}$, equation (4.1.6) shows that $v$ also lies in $\mathbb{Q}_{t}^{r}$. Since $M$ has a full set of independent solutions in this space, it follows that we may solve the equation $M y=v$ and find a 
solution $y$ in $\mathbb{Q}_{t}^{r}$. Thus by (4.1.5), $y \in \mathbb{Q}_{t}^{r} \cap \operatorname{ker}_{t} L=\operatorname{ker}_{t} M$ and hence $v=M y=0$, a contradiction which proves the injectivity of (4.1.3) as asserted. The theorem now follows from Theorem 3.1.6.

4.2 Let $b$ be a real number, $b \in(0,1)$ and let $\Delta$ be the annulus $D\left(0,1^{-}\right)-$ $D\left(0, b^{-}\right)$. Let $L \in \Re_{\Delta}$. Let $M$ be a monic element of $\Re$ defined by

$$
\operatorname{ker}_{t} M=\mathfrak{Q}_{t} \cap \operatorname{ker}_{t} L
$$

We have shown in 4.1 that $M \in \Re_{\Delta^{\prime}}$ where $\Delta^{\prime}$ is the annulus $D\left(0,1^{-}\right)$ - $D\left(0, b^{\prime-}\right)$ for some $b^{\prime} \in[b, 1)$.

COMPARISON THEOREM.

$$
\operatorname{dim}\left(\operatorname{ker}_{t} L \cap \mathbb{Q}_{t}\right) \geqslant \operatorname{dim}\left(\operatorname{ker} L \cap \mathbb{Q}_{\Delta}\right) \geqslant \operatorname{dim}\left(\operatorname{ker} L \cap \mathbb{Q}_{0}\right) .
$$

If equality holds then $M \in \Re_{A}$ where $A=D\left(0,1^{-}\right)$. Conversely if either $M \in \mathcal{S}_{A}$ or both $M \in \Re_{A}$ and $L \in \widetilde{S}_{A}$, then equality holds.

Note. "Equality" is used in the sense of both inequalities being replaced by equalities.

PROOF. If $u \in \mathbb{Q}_{\Delta}, L u=0$ then $M u$ is certainly a meromorphic function on $\Delta^{\prime}$ which lies in the kernel of $N$. If $M u \neq 0$ then by Theorem $2.5, N$ has nontrivial kernel in $\mathfrak{Q}_{t}$ which contradicts the definition of $M$. This shows that the right side of equation (4.2.2) is bounded by the order of $M$ which completes the proof of equation (4.2.2).

If equality holds in (4.2.2) then trivially the coefficients of $M$ are meromorphic functions on $D\left(0,1^{-}\right)$as well as being analytic elements on $\Delta^{\prime}$. It follows that there exists a polynomial $P$ with coefficients in $K$ such that $P M$ has coefficients in $H(A)$, i.e. $M \in \Re_{A}$.

Conversely if $M$ (which is by hypothesis monic) has coefficients in $H(A)$ then letting $s$ be the order of $M$, we have for each integer $m \geqslant 0$,

$$
D^{m} / m ! \equiv \sum_{j=0}^{s-1} B_{m, j} D^{j} \bmod \Re M
$$

where each $B_{m, j} \in H(A)$. Since $\operatorname{ker}_{t} M \subset \mathbb{Q}_{t}$ it is clear that for $0 \leqslant j<s$, and $r<1$

$$
\lim _{m \rightarrow \infty}\left|B_{m, j}\right|_{E} r^{m}=0 .
$$

Hence by the maximum modulus theorem

$$
\lim _{m \rightarrow \infty}\left|B_{m, j}(0)\right| r^{m}=0 .
$$

This shows that the kernel of $M$ at zero lies in $\mathscr{Q}_{0}$, and this proves the first part of the converse. If $L \in \mathfrak{S}_{A}$ and $M \in \Re_{A}$ then $M$ can have only removable 
singularities. By definition $\operatorname{ker}_{t} M$ lies in $\mathbb{Q}_{t}$ and hence by Lemma 4.2 .7 below the germs at zero annihilated by $M$ lie in $\mathbb{Q}_{0}$ which proves the second part of the converse.

4.2.6 Corollary. If $L \in \Re_{\Delta}, L$ of order $n$ and the equation $L u=0$ has $n$ independent solutions in $\mathfrak{Q}_{0}$ then these solutions lie in $W_{0}^{1, n-1}$.

(This confirms Conjecture 2 of [3].)

4.2.7 Lemma. If $A=D\left(0,1^{-}\right), L \in \Re_{A}$ and $L$ has only removable singularities in $A$ (in the strict sense that for each $\alpha \in A$ there exist $n$ linearly independent germs of holomorphic functions at $\alpha$ in the kernel of $L$ ) and if $\operatorname{ker}_{t} L$ lies in $\mathfrak{Q}_{t}$, then

$$
\operatorname{Ker}_{0} L \subset W_{0}^{1, n-1} .
$$

Proof OF 4.2.6 AND 4.2.7. The theorem shows that the hypothesis of 4.2.6 implies the hypothesis of Lemma 4.2.7. The proof of Lemma 4.2.7 for $L \in \Re_{0}$ may be found in Lemma 4.25 of [11]. The same proof is valid under the weaker hypothesis that $L \in \Re_{A}$. This completes the proofs of 4.2.6 and 4.2.7.

4.2.8 The statement of the comparison theorem may be sharpened by replacing the right hand side of (4.2.2) by $\operatorname{dim} \operatorname{mer} \operatorname{ker}_{A} L$. We omit the proof which merely repeats the proof of the given assertion.

4.2.9 Let $A$ be a standard set, let $L$ be an element of $\Re_{A}$ with factorization in $\Re$ given by equation (4.1.1) where

$$
\operatorname{ker}_{t} M \subset \mathbb{Q}_{t}, \quad \operatorname{ker}_{t} N \cap \mathbb{Q}_{t} \neq\{0\} .
$$

Then the tangential map (4.1.3) is not injective on $\left(\mathbb{Q}_{t}[D]\right)_{n-1} \times\left(\mathbb{Q}_{t}[D]\right)_{m-1}$. (And so the technique of this section gives no information if say $\operatorname{ker}_{t} L \subset \mathbb{Q}_{t}$, $\operatorname{ker}_{t} M=\left(\operatorname{ker}_{t} L\right) \cap W_{t}^{1,0}$.

Proof. Let $m$ be the order of $M$, let $u_{1}, \ldots, u_{m}$ be basis of $\operatorname{ker}_{t} M$ and let $v$ be a nonzero element of $\operatorname{ker}_{t} N \cap \mathbb{Q}_{t}$. The wronskian of $\left(u_{1}, \ldots, u_{m}\right)$ is nonzero in $D\left(t, 1^{-}\right)$and clearly lies in $Q_{t}$. Hence we may choose $Q$ in $\left(Q_{t}[D]\right)_{m-1}$ such that $Q\left(u_{i}\right)=0,1=1,2, \ldots, m-1, Q\left(u_{m}\right)=v$. Clearly $N \circ Q$ annihilates $\operatorname{ker}_{t} M$ and hence there exists $P \in\left(Q_{t}[D]\right)_{n-1}$ such that $(P, Q)$ lies in the kernel of mapping (4.1.3).

4.3 Index on special sets. It was shown previously [11, §4.16] that if $L \in \Re_{0}$ is injective on $\mathscr{Q}_{t}$, then it has index on $\mathscr{Q}_{a}$ for each $a \in D\left(0,1^{+}\right)$. The object of this section is to extend this result in two directions. We eliminate the hypothesis that the coefficients of the differential operator be rational and we extend the results to the case of annuli.

4.3.1 Lemma. Let $\mathfrak{p}$ be an element of $\Omega[X]$ then for each special set $A$, multiplication by $\mathfrak{p}$ is an injective endomorphism of $W_{A}$ (resp. $\left.\dot{W}_{A}\right)$ which has a 
continuous left inverse $\sigma_{A}\left(\right.$ resp. $\left.\dot{\sigma}_{A}\right)$. The index is the negative of the number of zeros of $\mathfrak{p}$ in $A$ (resp. union of $A$ with its circumference). Given $\varepsilon$ there exists $e$ depending upon $\mathfrak{p}$ such that if $e(A)>e$ then

$$
\operatorname{Max}\left(\left\|\sigma_{A}\right\|,\left\|\dot{\sigma}_{A}\right\|\right) \leqslant\left|\mathfrak{p}^{-1}\right|_{E}+\varepsilon .
$$

Proof. If $\mathfrak{p}$ has no zero in $A$ then $1 / p$ is stable on $W_{A}$. It is also stable on $W_{A}$ if $\mathfrak{p}$ has no zero on the circumference of $A$. If $\alpha \in A(\alpha \in A \cup$ (circumference of $A)$ ) then $u \rightarrow(u-u(\alpha)) /(x-\alpha)$ is stable on $W_{A}$ (resp. $\left.W_{A}\right)$. As in [12] in the first case one estimates $\operatorname{Inf}_{x \in A}|\mathfrak{p}(x)|$ and in the second case one uses the local Taylor expansion of $u(x)-u(x)$ at $\alpha$ to estimate the norm of $\sigma_{A}$ and

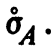

4.3.2 Proposition. Let $C$ be a special set. Let $\left\{B_{i}\right\}$ be a sequence of proper subsets of $C$ such that $B_{i}$ is a proper subset of $B_{i+1}$ for each $i \in \mathbf{N}$, and such that $C=\cup B_{i}$. Let $\theta$ be a continuous endomorphism of $\mathfrak{Q}_{C}$ such that for each $i \in \mathbf{N}$, the restriction of $\theta$ to $\mathscr{W}_{B_{i}}$ is a continuous endomorphism with index $\chi$, which is independent of $i$. Then $\theta$ has index as endomorphism of $\mathfrak{Q}_{C}$ which is equal to $\chi$.

Proof. The proof is the same as that of $[11, \S 4.7]$ since, for $j>i, \dot{W}_{B_{j}}$ is dense in $W_{B_{i}}$ and $Q_{C}=$ proj $\lim \dot{W}_{B_{i}}$.

4.3.3 Theorem. Let $A$ be a standard set, let $L$ be an element of $\Im_{A}$ which is injective on $\mathbb{Q}_{t}$. Then there exists $e \in(0,1)$ such that for each special set $B$ contained by $A$ with $e(B)>e$ we may conclude that $L$ is injective on $\mathbb{Q}_{B}$ and has index as endomorphism of $\mathfrak{Q}_{B}$.

Proof. With no loss in generality we may assume that $B$ does not lie in the infinite residue class and hence we may assume that $A$ has zero intersection with that class. By hypothesis there exists $Q \in \Re_{0}$ such that $\|Q L-1\|_{1,0}<1$. There exists $\mathfrak{p} \in K[x],|\mathfrak{p}|_{E}=1$ such that $P=\mathfrak{p} Q$ has coefficients in $K[x]$. Thus $\|P L-\mathfrak{p}\|_{1,0}<1$ and hence by Lemma 3.3 there exists $\varepsilon>0, e^{\prime} \in(0,1)$ such that if $B$ is a special set with $e_{B}>e^{\prime}$, we have

$$
\|P L-\mathfrak{p}\|_{\mathscr{W}_{B}}<1 /(1+\varepsilon) .
$$

On the other hand by Lemma 4.3.1 there exists $e^{\prime \prime} \in\left(e^{\prime}, 1\right)$ such that $e_{B} \geqslant e^{\prime \prime}$, $B$ special, implies that $\left\|\dot{\sigma}_{B}\right\|<1+\varepsilon$. Hence by $[11, \S 4.4] P L$ is injective and has index on $\dot{W}_{B}$ equal to the negative of the number of zeros of $\mathfrak{p}$ in the union of $B$ with its circumference.

We now choose $e^{\prime \prime \prime}$ so close to 1 that if $C$ is a special set with $e_{C}>e^{\prime \prime \prime}$ then there exists a sequence of proper subsets $B_{i}$ as in the first sentence of 4.3.2 such that the number of zeros of $\mathfrak{p}$ in $B_{i}$ is independent of $i$. We now let $e=\max \left(e^{\prime \prime}, e^{\prime \prime \prime}\right)$. If $C$ is a special set contained by $A$ with $e_{C}>e$ then we 
choose the sequence $\left\{B_{i}\right\}$ as in 4.3.2, conclude that the index of $P L$ in $\dot{W}_{B_{i}}$ is independent of $i$ which shows that $P L$ has index as endomorphism of $\mathscr{Q}_{C}$. By the same argument $P L$ is injective on $\mathbb{Q}_{C}$. Since $P$ is a differential operator, $P$ has finite dimensional kernel in $\mathbb{Q}_{C}$ and so $P$ has index on $\mathbb{Q}_{C}$ and thus the same holds for $L$ as asserted. Finally since $P L$ is injective the same holds for $L$.

4.4 Index theorem. Let $A=D\left(0,1^{-}\right)$, let $L \in \Re_{A}$. To discuss the index of $L$ as endomorphism of $\mathbb{C}_{0}$ we must insist that the coefficients of $L$ have no poles in $A$. Thus we impose the condition that $L \in \widetilde{S}_{A}$.

THEOREM. If $L \in S_{A}$ and (4.2.2) is an equality then $L$ has index as endomorphism of $\mathfrak{Q}_{0}$.

Proof. Let $M$ be defined by equation (4.2.1) so $M \in \mathbb{S}_{\Delta}$ for some superadmissible subset $\Delta$ of $A$, i.e. $\Delta$ is an annulus with center at zero and outer radius unity. It follows from Theorem 4.2 that $\mathfrak{R} \in \mathfrak{R}_{A}$. Thus again putting $L=N \cdot M, N$ is an element of $\Re_{A}$ and

$$
\mathbb{Q}_{t} \cap \operatorname{ker}_{t} N=\{0\} .
$$

Since $M$ need not be an element of $\widetilde{S}_{A}$ we first choose a polynomial $P$ with coefficients in $K$ such that $P \cdot M \in \widetilde{S}_{A}$ and then putting $M^{\prime}=P \cdot M$ we choose $Q \in K[x]$ such that

$$
Q L=N^{\prime} \cdot M^{\prime}
$$

where $N^{\prime}=Q N \cdot(1 / P) \in \Im_{A}$. Clearly $M^{\prime}$ annihilates a full set of independent elements of $\mathscr{Q}_{0}$ and $N^{\prime}$ is injective on $\mathbb{Q}_{t}$. Thus $M^{\prime}$ is a surjective mapping of $\mathscr{C}_{0}$ into itself and by Theorem 4.3.3 $N^{\prime}$ has index as endomorphism of $\mathbb{Q}_{0}$. This shows that $Q L$ and hence $L$ has index as asserted.

4.4.3 COROLLARY. Let $B$ be a very standard set and $L$ an element of $\Im_{B}$. Then for all but a finite number of residue classes $D\left(a, 1^{-}\right)$lying in $B, L$ has index as endomorphism of $\mathfrak{Q}_{a}$.

Proof. Let $M$ be defined by (4.2.1). We know that $M \in \widetilde{S}_{B^{\prime}}$ for some superadmissible subset $B^{\prime}$ of $B$. Hence $M \subset \subseteq_{C}$ where $C$ is an admissible very standard subset of $B$. If $A=D\left(a, 1^{-}\right)$is a residue class lying in $C$ then by Theorem 4.2 equality holds in (4.2.2) and hence by the present theorem $L$ has index as endomorphism of $\mathbb{Q}_{a}$. This completes the proof of the corollary.

5. Approximate rational solutions of linear differential equations. The object of this section is to show that if $L \in \Re$ then $L$ has kernel in $W_{t}^{1,0}$ if and only if the equation $L u=0$ has approximate solutions in $E$. This may be useful in understanding the limitations of Theorem 3.1.6. 
5.1 Lemma. If $L \in \Re$ then $\|L\|_{1,0}=|L|_{E}$.

Proof. Clearly $W_{t}^{1,0} \subset E$ which gives the inequality in one direction but in fact by $[11, \S 1.11 .5]$ there exists a polynomial $u$ such that $\|L\|_{W_{1}^{1,0}}$ $=|L u|_{E} /|u|_{E}$. This gives the inequality in the other direction.

$5.2 \mathrm{It}$ is known that if $L$ is injective on $W_{t}^{1,0}$ then $L$ is invertible [11, Proposition 4.20].

Lemma. Let $L$ be injective on $W_{t}^{1,0}$. Then $L$ is invertible on both $W_{t}^{1,0}$ and $E$ and $\left\|L^{-1}\right\|_{1,0}=\left|L^{-1}\right|_{E}$.

Proof. Since $L$ is injective on $W_{t}^{1,0}$ we know by [11, Theorem 2.6] that there exists $Q \in \Re_{0}$ such that $\|Q L-1\|_{1,0}<1$. Thus $|Q L-1|_{E}<1$. This shows that $Q L$ is invertible as endomorphism of $E$ and as endomorphism of $W_{t}^{1,0}$ and hence $Q$ is surjective on $E$ and on $W_{t}^{1,0}$. It follows from [11, 4.10] that $Q$ is injective on $W_{t, 0}^{1,0}$ and hence on $E$. Thus by Proposition 3.4.6, $L$ is invertible on $E$ and on $W_{t}^{1,0}$. Now $E$ being a subspace of $W_{t}^{1,0}$ with the induced norm, $\left|L^{-1}\right|_{E} \leqslant\left\|L^{-1}\right\|_{1,0}$. Now

$$
\left|Q-L^{-1}\right|_{E}=\left|(Q L-1) L^{-1}\right|_{E}<\left|L^{-1}\right|_{E}
$$

and hence

$$
\left|L^{-1}\right|_{E}=|Q|_{E}=\|Q\|_{1,0}=\left\|L^{-1}\right\|_{1,0}
$$

since

$$
\left\|Q-L^{-1}\right\|_{1,0}=\left\|(Q L-1) L^{-1}\right\|_{1,0}<\left\|L^{-1}\right\|_{1,0} .
$$

This completes the proof.

5.3 Lemma. Let $L$ be monic element of $\Re$ of order $n$. Then given $\varepsilon<0$ there exists $M$ monic element of $\Re$ of order $n$ such that

$$
\|L-M\|_{1,0}<\varepsilon
$$

and such that $M$ is injective on $W_{t}^{1,0}$.

Proof. If $n=1$ then we may suppose that $L=D-\alpha$ where $\alpha=u^{\prime} / u, u$ $\in W_{t}^{1,0},\|u\|_{1,0}=1$. We choose $s \in \mathbf{N}$ such that $\left|p^{s}\right|<\varepsilon$ and let

$$
M=D-\alpha-p^{s} x^{p^{s}-1} .
$$

The kernel of $M$ at $t$ is spanned by $u \exp \left(x^{p^{s}}-t^{p^{s}}\right)$ which does not lie in $\mathbb{Q}_{t}$. This proves the assertion for $n=1$ and we now proceed by induction on $n$.

If $n>1$, let 


$$
L=D^{n}+a_{n-1} D^{n-1}+\cdots+a_{0} .
$$

We may by the case $n=1$ choose $b \in E$ such that

$$
\left|b-a_{n-1}\right|<\varepsilon
$$

but the wronskian at $t$ of

$$
R=D^{n}+b D^{n-1}+a_{n-2} D^{n-2}+\cdots+a_{0}
$$

does not lie in $\mathscr{Q}_{t}$. Thus the kernel at $t$ of $\Re$ does not lie in $\mathfrak{Q}_{t}$. Thus by [11, Theorem 2.6],

$$
R=N \cdot M_{1}, \quad \operatorname{ker}_{t} M_{1}=\mathbb{Q}_{t} \cap \operatorname{Ker}_{t} R, \quad \operatorname{order} M_{1}<n .
$$

By induction on $n$ there exists $Q \in \Re$ of the same order as $M_{1}$ such that

$$
\left\|M_{1}-Q\right\|_{1,0}<\varepsilon /\|N\|_{1,0}
$$

and such that $Q$ is injective on $W_{t}^{1,0}$. We now put $M=N \cdot Q$ which certainly satisfies (5.3.1). If now $u \in W_{t}^{1,0}, M u=0$, then $Q u$ is not zero but does lie in $W_{t}^{1,0}$ which shows that $N$ is not injective in $W_{t}^{1,0}$ and hence contradicting the definition of $M_{1}$. This completes the proof of the lemma.

5.4 THEOREM. Let $L \in \Re$. Then $L$ has nontrivial kernel in $W_{t}^{1,0}\left(\right.$ or in $\left.Q_{t}\right)$ if and only if there exists a sequence $\left\{v_{n}\right\}, v_{n} \in E_{0}$, such that $\left|v_{n}\right|=1$ for all $n$ but

$$
L v_{n} \rightarrow 0
$$

in $E$.

Proof. If $L$ is injective on $W_{t}^{1,0}$ then by 5.2, $L$ is invertible in $E$ and thus equation (5.4.1) implies

$$
1=\left|v_{n}\right|_{E}=\left|L^{-1}\left(L v_{n}\right)\right|_{E} \leqslant\left|L^{-1}\right|_{E}\left|L v_{n}\right|_{E} \rightarrow 0
$$

which is impossible. Thus the existence of the sequence implies that $L$ is not injective on $W_{t}^{1,0}$.

Conversely let $L$ have a nontrivial kernel in $W_{t}^{1,0}$. Then by Lemma 5.3 there exists a sequence $\left\{L_{n}\right\}$ oi elements of $\Re$ which are injective on $W_{t}^{1,0}$ and such that

$$
\left\|L-L_{n}\right\|_{1,0} \rightarrow 0 .
$$

By Lemma 5.2, $L_{n}$ is invertible on both $W_{t}^{1,0}$ and $E$. We assert that

$$
\left\|L_{n}^{-1}\right\|_{1,0} \rightarrow \infty \text {. }
$$


Otherwise there exists $c$ such that for all $n \in \mathbf{N},\left\|L_{n}^{-1}\right\|_{1,0}<c$. Hence

$$
\left\|1-L_{n}^{-1} L\right\|_{1,0}=\left\|L_{n}^{-1}\left(L_{n}-L\right)\right\|_{1,0} \leqslant c\left\|L_{n}-L_{0}\right\|_{1,0}
$$

which by (5.4.3) shows that there exists $n$ such that $\left\|1-L_{n}^{-1} L\right\|_{1,0}<1$ and hence there exists $B$, continuous endomorphism of $W_{t}^{1,0}$ such that $B L_{n}^{-1} L=I$, and hence $L$ is injective, contrary to hypothesis.

This proves (5.4.4). This means by Lemma 5.2 that there exists a sequence $\left\{u_{n}\right\}$ in $E$ such that $\left|u_{n}\right|_{E}=1, L_{n} u_{n} \rightarrow 0$. If now $\left\{v_{n}\right\}$ is a sequence in $E_{0}$ such that $\left|u_{n}-v_{n}\right|_{E} \rightarrow 0$, then for all $n$ large, we have $\left|v_{n}\right|_{E}=1$ while clearly $L v_{n} \rightarrow 0$. This completes the proof of the theorem.

6. Differential equations over the residue class field. Let $A$ be a standard set and let $\Re_{A}$ be the ring of linear differential operators whose coefficients are ratios of elements of $H(A)$. Our object is to study factorization in $\Re_{A}$ by passing to the residue class field. We therefore first recall some well-known results for characteristic $p$.

6.1 Let $\bar{K}$ be the residue class field of $K, \bar{E}_{0}=\bar{K}(X)$, let $\bar{\Re}_{0}=\bar{E}_{0}[D]$, the ring of differential operators with coefficients in $\bar{E}_{0}$. Elements of $\bar{\Re}_{0}$ act as (but are not identified with) linear transformations of $\bar{E}_{0}$ as vector space over $\bar{K}\left(X^{p}\right)$. The element $D^{p}$ annihilates $\bar{E}_{0}$ and also lies in the center of $\bar{\Re}_{0}$. The ring $\bar{\Re}_{0}$ has both right and left division algorithm and in particular left ideals are principal.

6.1.1 Let $u_{1}, \ldots, u_{m}$ be elements of $\bar{E}_{0}$ linearly independent over $\bar{K}\left(X^{p}\right)$; then by a classical proof $[9$, p. 10$]$, the wronskian $W\left(u_{1}, \ldots, u_{n}\right)$ is a nonzero element of $\bar{E}_{0}$. An immediate consequence is that if $L$ is an element of $\bar{\Re}_{0}$ of order $m$, then $\operatorname{ker} L$, the kernel of $L$ in $\bar{E}_{0}$, has dimension at most $m$ over $\bar{K}\left(X^{p}\right)$.

6.1.2 Lemma. Let $L$ be an element of $\bar{\Re}_{0}$ of order $m$. Then

$$
\operatorname{dim}_{\bar{K}\left(X^{p}\right)} \operatorname{ker} L=m
$$

if and only if $D^{p}$ lies in the ideal $\bar{\Re}_{0} L$.

Proof. By the division algorithm there exist elements $A, B$ of $\bar{\Re}_{0}$ such that order $B \leqslant m-1$ and such that $D^{p}=A L+B$. Since $D^{p}$ annihilates $\bar{E}_{0}, B$ must annihilate $\operatorname{ker} L$. Hence by 6.1.1 if $\operatorname{ker} L$ has dimension $m$ over $K\left(X^{p}\right)$ then $B=0$. Conversely if $B=0$, then $A L$ annihilates $\bar{E}_{0}$ and hence $L \bar{E}_{0}$ lies in the kernel of $A$. Thus

$$
\operatorname{dim}_{\bar{K}\left(X^{p}\right)} L \bar{E}_{0} \leqslant \operatorname{order} A=p-m
$$

which shows that 


$$
\operatorname{dim}_{\bar{K}\left(X^{p}\right)} \operatorname{ker} L \geqslant m .
$$

Equality now follows from 6.1.1 which completes the proof.

6.1.2.1 CoROLlary. Let $M$ be the generator of the left ideal generated by $D^{p}$ and $L$, i.e.

$$
\bar{\Re}_{0} D^{p}+\bar{\Re}_{0} L=\bar{\Re}_{0} M,
$$

then $\operatorname{ker} L=\operatorname{ker} M$ and the dimension of $\operatorname{ker} M$ coincides with the order of $M$.

Proof. Since $D^{p} \in \bar{\Re}_{0} M$, the dimension of $\operatorname{ker} M$ is equal to its order. Since $L$ is a left multiple of $M, \operatorname{ker} L$ contains $\operatorname{ker} M$. Since there exist $A, B \in \bar{\Re}_{0}$ such that $A D^{p}+B L=M$, ker $M$ contains $\operatorname{ker} L$.

6.1.2.2 CoRollaRY. If $\operatorname{ker} L=\{0\}$ then $\bar{\Re}_{0} D^{p}+\bar{\Re}_{0} L=\bar{\Re}_{0}$ and conversely.

6.1.2.3 CoRollary. If $L, N \in \bar{\Re}_{0}$ then $\operatorname{ker} L \subset \operatorname{ker} N$ if and only if

$$
N \in \bar{\Re}_{0} D^{p}+\bar{\Re}_{0} L .
$$

Proof. Let $M$ be the generator of the ideal on the right. If $N=B M$ then $\operatorname{ker} N \supset \operatorname{ker} M=\operatorname{ker} L$. Conversely given $N \in \bar{\Re}_{0}$ we may choose $A, B$ $\in \bar{\Re}_{0}$, order $B<\operatorname{order} M$ such that $N=A M+B$. Hence $\operatorname{ker} N \supset \operatorname{ker} L=$ ker $M$ implies that $\operatorname{ker} B$ contains ker $M$ and hence by $6.1 .1, B=0$.

6.1.3 Now let $L$ be an $n \times n$ matrix with coefficients in $\bar{\Re}_{0}$. Hence $L$ acts on $\bar{E}_{0}^{n}$, the $\bar{K}\left(X^{p}\right)$ space of $n$-tuples with coefficients in $\bar{E}_{0}$.

LEMMA. If $L$ is injective as endomorphism of $\bar{E}_{0}^{n}$ then there exist $n \times n$ matrices, $Q, H$ with coefficients in $\bar{\Re}_{0}$ such that

$$
Q L+H D^{p}=I
$$

Proof. By the theory of elementary divisors there exist $V, U$, invertible $n \times n$ matrices with coefficients in $\bar{\Re}_{0}$ such that $V L U$ is diagonal matrix with coefficients $\varepsilon_{1}, \ldots, \varepsilon_{n}$. By hypothesis $L$ is injective and hence each $\varepsilon_{j}$ is injective on $\bar{E}_{0}$. Thus by 6.1 .2 .2 there exist $A_{j}, B_{j} \in \bar{\Re}_{0}$ such that $A_{i} \varepsilon_{i}+B_{i} D^{p}$ $=1, i=1,2, \ldots, n$. Let now $A$ (resp. $B$ ) be the $n \times n$ diagonal matrix whose diagonal entries are $\left(A_{1}, \ldots, A_{n}\right)$ (resp. $\left.\left(B_{1}, \ldots, B_{n}\right)\right)$. Then $A V L U+B D^{p}$ $=I$. The assertion follows by multiplying on the left by $U$, on right by $U^{-1}$ and setting $Q=U A V, H=U B U^{-1}$. This completes the proof.

6.1.3.1 Corollary. An injective differential endomorphism of $\bar{E}_{0}^{n}$ has inverse which is also a differential endomorphism.

(Explanation: By a differential endomorphism of $\bar{E}_{0}^{n}$, we mean an endomor- 
phism of $\bar{E}_{0}^{n}$ as $\bar{K}\left(X^{p}\right)$ space given by an $n \times n$ matrix with coefficients in $\Re_{0}$.)

6.2 The residue class field of $E$ coincides with $\bar{E}_{0}=\bar{K}(X)$. The natural mapping of the valuation ring, $\mathfrak{N}_{E}$, of $E$ into $\bar{E}$ may be extended to a map

$$
L \mapsto \bar{L}
$$

of $\mathfrak{D}_{E}[D]$ into $\bar{E}_{0}[D]=\bar{\Re}_{0}$. We intend to obtain information about factorization of elements, $L$, of $\Re$ from corresponding information about $\bar{L}$. We first consider an analogue of Theorem 3.1.6.

6.2.1 Let $F$ be given by equation (3.1.1) and be subject to the further condition that $m=s$ and that each $C_{\nu, \mu}$ maps $A$ into $D\left(0,1^{+}\right)$, i.e.

$$
\left|C_{\nu, \mu}\right|_{E} \leqslant 1 \quad \forall \nu, \mu
$$

and furthermore we insist that

$$
\left|C_{\nu, \mu}\right|_{E} \rightarrow 0 \text { as }(\nu, \mu) \rightarrow \infty .
$$

Then we define $\bar{F}$ to be the image of $F$ in the ring of $s \times s$ matrices with coefficients in $\bar{E}_{0}[X, Y]$. If $u=\left(\bar{u}_{1}, \ldots, \bar{u}_{s}\right) \in \bar{E}_{0}^{s}$ then we define $\bar{L}_{\bar{u}}$, the reduced tangential mapping at $\bar{u}$, to be the differential operator (on $z$ $\left.=\left(z_{1}, \ldots, z_{s}\right)\right)$ obtained from equation (3.1.4) by mapping coefficients into $\bar{E}_{0}$.

6.2.1 THEOREM. Let $F$ be given by equation (3.1.1) subject to conditions (6.2.1.1) and (6.2.1.2). Let $\bar{u} \in \bar{E}_{0}^{s}$ be a solution of the reduced system

$$
\bar{F}(\bar{u}, d \bar{u} / d x)=0
$$

and suppose that the reduced tangential mapping, $\bar{L}_{\bar{u}}$, is injective on $\bar{E}_{0}^{s}$. Then $\bar{u}$ lifts uniquely to a solution, $u$, in $E^{s}$ of equation (3.1.3) (and hence (by Theorem 5.4) $u$ satisfies the conditions of Theorem 3.1.6 and therefore $u \in H(B)^{s}$ for some superadmissible subset $B$ of $A$ ).

Proof. Let $\eta$ be an arbitrary lifting of $\bar{u}$ to $\bigcap_{E_{0}}^{s}$. By Theorem 5.4, $L_{\eta}$ is injective on $\left(W_{t}^{1,0}\right)^{s}$ and hence by Lemma 3.4 there exists an $s \times s$ matrix $Q$ with coefficients in $\Re_{0}$ such that

$$
\left\|Q L_{\eta}-I\right\|_{E}=\left\|Q L_{\eta}-I\right\|_{1,0}<1 .
$$

It follows from this relation, from [11, §4.10] and from Proposition 3.4.6 that $L_{\eta}$ is invertible in $E$. We assert that

$$
\left\|L_{\eta}^{-1}\right\|_{E}=1
$$

The inequality in one direction is given by $1=\|I\|=\left\|L_{\eta}^{-1} \circ L_{\eta}\right\|$ 
$\leqslant\left\|L_{\eta}^{-1}\right\|$. The inequality in the other direction is obtained by supposing otherwise then there would exist $w, z \in E^{s}$ such that $|w|_{E}=1,|z|_{E}<1$ for which $L_{\eta}^{-1} z=w$. Hence $z=L_{\eta} w$ which would imply that $\bar{L}_{\bar{u}}=\bar{L}_{\eta}$ annihilates the nontrivial element $\bar{w}$ of $\bar{E}_{0}^{s}$ contrary to hypothesis. This completes the verification of 6.2.1.5.

We now let $\lambda=F(\eta, d \eta / d x)$, so by equation (6.2.1.3), there exists $\varepsilon$ such that

$$
|\lambda|_{E}<\varepsilon<1 .
$$

Let $U=\left\{\left.z \in E^{s}|| z\right|_{E} \leqslant \varepsilon\right\}$. Let $\phi$ be given by equation (3.6.1); then $\phi$ is a contractive map of $U$ into itself and hence has a unique fixed point, $z_{\phi}$. Thus $u=\eta+z_{\phi}$ is a solution of equation (3.1.3) and $u$ lies above $\bar{u}$ since $z_{\phi} \in U$. If $v$ is any other solution of equation (3.1.3) above $\bar{u}$, then

$$
|v-\eta|=\varepsilon^{\prime}<1 .
$$

Replacing $\varepsilon$ by $\operatorname{Max}\left(\varepsilon, \varepsilon^{\prime}\right)$, it is clear that $v=u$. This completes the proof of the theorem.

6.2.2 (i) We now formulate a Hensel lemma for elements of $\Re$. For this purpose let $\bar{\Re}_{0}^{[h]}\left(\right.$ resp. $\left.\Re^{[h]}\right)$ denote (for each $h \in \mathrm{N}$ ) the set of all elements of $\bar{\Re}_{0}$ (resp. $\Re$ ) of order not greater than $h$.

THEOREM 6.2.2. (i) Let $L$ be an element of $\mathfrak{D}_{E}[D]$ of order $n+m$ and have image, $\bar{L}$, in $\bar{\Re}_{0}$ which has a right monic factor, $\bar{M}$, of order $m$, i.e.

$$
\bar{L}=\bar{N} \circ \bar{M}
$$

(so $n \geqslant \operatorname{order} \bar{N}$ ). Let $\bar{L}_{\bar{N}, \bar{M}}$ be the mapping

$$
\bar{L}_{\bar{N}, \bar{M}}:(\bar{P}, \bar{Q}) \rightarrow \bar{P} \bar{M}+\bar{N} \bar{Q}
$$

of $\bar{\Re}_{0}^{[n-1]} \times \bar{\Re}_{0}^{[m-1]}$ into $\bar{\Re}_{0}^{[n+m-1]}$. If this mapping is injective then $\bar{M}$ lifts to a unique monic right divisor, $M$, of $L$ of order $m$ in $\Re$ and hence $L=N \circ M$ where $N$ is also in $\Re$ and of order $n$.

(ii) Under the hypothesis of (i) if $L \in \Re_{A}$ then $N$ and $M$ lie in $\Re_{B}$ for some super admissible subset $B$ of $A$.

(iii) Under the hypothesis of (i) we consider the explicit description of $\bar{L}_{\bar{N}, \bar{M}}$. As $\bar{E}_{0}$ space $\bar{\Re}_{0}^{[n-1]} \times \bar{\Re}_{0}^{[m-1]}$ has basis consisting of all pairs $\left(D^{i}, 0\right)$ and $\left(0, D^{j}\right)$ where $0 \leqslant i<n, 0 \leqslant j<m$ and $\bar{\Re}_{0}^{[n+m-1]}$ has basis consisting of $\left\{D^{i}\right\}_{0 \leqslant i<n+m}$. Now $\bar{L}_{\bar{N}, \bar{M}}$ is a linear mapping of $\bar{K}\left(X^{p}\right)$ space, not of $\bar{E}_{0}$ space, but using the indicated bases, $\bar{L}_{\bar{N}, \bar{M}}$ may be represented by an $(n+m) \times(n+$ m) matrix, $\left(\bar{L}_{\bar{N}, \bar{M}}\right)$, with coefficients in $\bar{\Re}_{0}$ and by Lemma 6.1 .3 and the hypothesis of injectivity there exists an "inverse" matrix, $\bar{J}$, with coefficients in $\bar{\Re}_{0}$ such that 


$$
\bar{J} \circ\left(\bar{L}_{\bar{N}, \bar{M}}\right) \equiv I \bmod \Re_{0} D^{p} .
$$

We assert that under hypothesis (ii), the set $B$ contains all residue classes lying in $A$ except possible for poles of $\bar{L}$, of $\bar{M}$ and of the coefficients in the differential operators appearing as coefficients of $\bar{J}$.

Proof. Equation (6.2.2.3) may be interpreted as a nonlinear differential equation in the coefficients of $N$ and $M,(6.2 .2 .1)$ is then the reduced equation and $\bar{L}_{\bar{N}, \bar{M}}$ is the reduced tangential map. Parts (i) and (ii) then follow from Theorem 6.2.1.

For the proof of (iii) let $N_{0}, M_{0}$ be liftings of $\bar{N}$ and $\bar{M}$ to $\Re_{0}$ such that $M_{0}$ is monic of order $m$ with coefficients having poles only in the residue classes corresponding to poles of $\bar{M}$ and let $N_{0}$ be of order not greater than $n$ and with poles corresponding to the poles of $\bar{N}$. We lift $\left(\bar{L}_{\bar{N}, \bar{M}}\right)$ to $\left(L_{N_{0}, M_{0}}\right)$ the matrix of $L_{N_{0}, M_{0}}$ viewed as mapping of $\Re_{0}^{[n-1]} \times \Re_{0}^{[m-1]}$ into $\Re_{0}^{[n+m-1]}$ using "bases" as above. Let $J$ be a lifting of $\bar{J}$ to an $(n+m) \times(n+m)$ matrix with coefficients in $\Re_{0}$ having poles only in the residue classes of poles of coefficients of $J$. Since $\left\|D^{p}\right\|_{1,0}<1$ we conclude from (6.2.2.3) that

$$
\left\|J \cdot\left(L_{N_{0}, M_{0}}\right)-I\right\|_{1,0}<1 .
$$

Thus $J$ plays the role of $Q$ in equation (3.4.1) and the assertion now follows from (3.6.5).

(6.2.3) We examine the main hypothesis of the previous theorem.

6.2.3.1 LemMa. Let $m, n$ be integers, let $\bar{M}$ (resp. $\bar{N})$ be element of $\bar{\Re}_{0}$ of order $m$ (resp. of order not greater than $n$ ). The mapping $\bar{L}_{\bar{N}, \bar{M}}$ defined by (6.2.2.2) is

(i) not injective if $\operatorname{ker} \bar{N}$ is not trivial while $\operatorname{ker} \bar{M}$ is of dimension $m$ over $\bar{K}\left(X^{p}\right)$ (in particular then if $\bar{M}=D^{m}$ while $\bar{N}$ has nontrivial kernel);

(ii) injective if $\operatorname{ker} \bar{N}=\{0\}, \operatorname{dim} \operatorname{ker} \bar{M}=m$;

(iii) injective if $\bar{N}$ is of zero order.

The proofs are self evident (the proof of (i) being a minor modification of the argument of \$4.2.9), and hence are omitted.

THEOREM 6.2.3. Let $A$ be a standard set and let $L$ be an element of $\Re_{A} \cap \mathfrak{D}_{E}[D]$ of order $n+m$ whose image, $\bar{L}$, in $\bar{\Re}_{0}$ is monic of order $m$. Then $\bar{L}$ has unique monic lifting $M$ of order $m$ in $\Re$ which divides $L$ on the left, i.e. $L=N \circ M$ and $M \in \Re_{B}$ where $B$ is a superadmissible subset of $A$ containing all residue classes in $A$ except those containing poles of the coefficients of $L$.

Proof. We apply Theorem 6.2.2 with $\bar{N}=1, \bar{M}=\bar{L}$. In the preceding lemma we noted that mapping (6.2.2.2) is injective. Thus it only remains to consider the matrix of the inverse of $\bar{L}_{\bar{N}, \bar{M}}$. The mapping $\bar{L}_{\bar{N}, \bar{M}}$ is of the form 


$$
(\bar{P}, \bar{Q}) \mapsto \overline{P M}+\bar{Q}
$$

The inverse mapping,

$$
\bar{S}=\overline{P M}+\bar{Q} \mapsto(\bar{P}, \bar{Q})
$$

is given by division of $\bar{S}$ by $\bar{M}$, a process which introduces no poles (other than those occuring in $\bar{M}$ ) since $\bar{M}$ is monic. This completes the proof of the theorem.

6.2.3.3 We may associate a Newton polygon with each element of $L$ of $\Re$ by viewing $L$ as a polynomial in $D$ with coefficients in the valued field $E$. The previous lemma asserts that there is a factor of $L$ in $\Re$ of order equal to the length of the projection of the first side of the Newton polygon of $L$ provided that side has negative or zero slope and provided that the second side has strictly positive slope. Similar results may be obtained if both the first side and the second side have strictly positive slopes. No assertion is made if the first side has strictly negative slope and the second side has zero slope (cf. (i) Lemma 6.2.3.1).

6.2.4 We now consider a second order differential equation. In view of the previous section we may assume that the leading term has maximal coefficient. Thus let

$$
L=D^{2}+a D+b
$$

be an element of $\Re_{A} \cap \mathfrak{D}_{E}[D]$ where $A$ is a standard set. Let

$$
\bar{L}=D^{2}+\bar{a} D+\bar{b}
$$

be the reduced operator and let the Riccati equation be written

$$
\eta^{2}+\eta^{\prime}+a \eta+b=0
$$

and the reduced form is

$$
\bar{\eta}^{2}+\overline{\eta^{\prime}}+\overline{a \eta}+\bar{b}=0 .
$$

We know that $L$ has a right factor, $D-\eta$

$$
L=(D+a+\eta)(D-\eta)
$$

if and only if $\eta$ is a solution of (6.2.4.2).

If $\bar{L}$ has a solution $\bar{u}$ in $\bar{E}_{0}$ then

$$
\bar{\eta}=\overline{u^{\prime}} / \bar{u}
$$

is a solution of $\left(6 \cdot 2 \cdot 4 \cdot 2^{\prime}\right)$. 
If however, $\bar{\eta}$ is a solution of $\left(6.2 .4 .2^{\prime}\right)$ as will now be assumed, no element $\bar{u}$ in $\bar{E}_{0}$ satisfying (6.2.4.4) need exist. Viewing $\left(6.2 .4 .2^{\prime}\right)$ as a nonlinear differential equation, the tangential mapping is

$$
z \mapsto(D+(\bar{a}+2 \bar{\eta})) z
$$

and if this is injective then by 6.2 .2 , the factorization

$$
\bar{L}=(D+\bar{a}+\bar{\eta})(D-\bar{\eta})
$$

lifts to a factorization in $\Re$ and indeed in $\Re_{B}$ where $B$ is a superadmissible subset of $A$. The injectivity of (6.2.4.5) is determined by division,

$$
D^{p}=\bar{Q} \circ(D+\bar{a}+2 \bar{\eta})+\bar{b}_{p},
$$

where $\bar{b}_{p} \in \bar{E}_{0}$ and $\bar{Q} \in \bar{\Re}_{0}$. Injectivity is equivalent to the nonvanishing of $\bar{b}_{p}$. Summarizing and using 6.2.2 we obtain:

LEMMA 6.2.4. If $\bar{b}_{p} \neq 0$ then there exists a unique lifting $\eta$ of $\bar{\eta}$ which satisfies (6.2.4.2) and which lies in $H(B)$ where $B$ is a superadmissible subset of $A$ containing all residue classes of $A$ except for the poles of $\bar{a}, \bar{b}$ and the zeros of $\bar{b}_{p}$.

The calculation of $\bar{b}_{p}$ can be achieved by setting $c=\bar{a}+2 \bar{\eta}$ and using the formula,

$$
-\bar{b}_{p}=c^{p}+D^{p-1} c .
$$

If there exists $\bar{u}$ satisfying (6.2.4.4), then we may replace (6.2.4.5) by $D+\bar{a}$, the differential operator for the wronskian of $\bar{L}$. In particular this section (6.2.4) gives no information if for example $\bar{a}=0$ and there exists $\bar{u}$ satisfying (6.2.4.4).

7. Nonexistence of index. It has been conjectured [11, §4.27] that if $L \in \Re_{0}$ and has say polynomial coefficients and if for each $y \in D\left(0,1^{-}\right)$there exists $r>0$ such that $L$ has index as endomorphism of $\mathbb{Q}_{0}^{r}$ then we may conclude that $L$ has index as endomorphism of $\mathfrak{Q}_{0}\left(=\mathbb{Q}_{0}^{1}\right)$. Thus it was conjectured that if $L$ has index on small disks then it has index on large disks.

In this section we give a counterexample to this conjecture. In our counterexample there is a lower bound for $r$ independent of $y \in D\left(0,1^{-}\right)$.

The confluent hypergeometric function

$$
\phi(a, c, x)=\sum_{s=0} \frac{(a)_{s} x^{s}}{(c)_{s} s !}
$$

which was brought to our attention by Monsky has been discussed in [11, \$4.26]. In particular it was noted (cf. [5]) that for $c=1 / p, a \in \mathbf{Z}_{p}, a \notin-\mathbf{N}$, 
this function satisfies a second order differential equation irreducible in $\Re_{0}$. Furthermore $\phi(a, 1 / p, x / p)$ lies in $\mathbb{Q}_{0}$ while the wronskian at $t$ of the corresponding differential equation has radius of convergence strictly less than unity. Thus Theorem 4.1 shows the existence of a factorization of that differential equation in $\Re_{A}$ where $A$ is a superadmissible subset of $\Omega$.

For further discussion we find it conveneint to replace $x$ by $1-x$, and to assume that $a \notin \mathbf{Z}$. Letting $D=d / d x$, we know that $\phi(a, 1 / p,(1-x) / p)$, is annihilated by

$$
L_{a}=p(1-x) D^{2}-x D-a .
$$

The counterexample will be given by $L_{a}$ where $a$ is a $p$-adic Liouville number satisfying condition (7.20) below.

In the following let $C$ be the special set

$$
C=D\left(0, b^{-}\right)-D\left(0, \beta^{+}\right)
$$

whose radii will be chosen later. Thus

$$
C=D\left(0, b^{-}\right) \cap C^{\prime}
$$

where $C^{\prime}=\{x|| x \mid>\beta\}$. In particular letting $\mathscr{Q}_{C^{\prime}}^{*}$ denote the elements of $\mathfrak{Q}_{C^{\prime}}$ which vanish at infinity,

$$
\mathbb{Q}_{C}=\mathbb{Q}_{0}^{b} \oplus \mathbb{Q}_{C^{\prime}}^{*}
$$

which simply expresses the decomposition of Laurent series into elements of $\Omega[[x]]$ and of $\Omega[[1 / x]] / x$. We observe that $L_{a}$ is stable on $\mathbb{Q}_{C}$, on $\mathbb{Q}_{0}^{b}$ and on $\mathbb{Q}_{C^{\prime}}^{*}$. We propose to choose $a$ so that $L_{a}$ has index on $\mathscr{Q}_{C}^{*}$, but not on $\mathfrak{Q}_{C}$. This then will show that $L_{a}$ does not have index on $\mathbb{Q}_{0}^{b}$.

7.4 Proposition. (i) A formal solution for $L_{a}$ is given by

$$
v=x^{-a} V
$$

where

$$
V=\sum_{m=0}^{\infty} \frac{1}{(-x)^{m}} B_{m}(a)_{m},
$$

the generating function, $u(\xi)=\sum_{m=0}^{\infty} B_{m} \xi^{m}$, for the $B_{m}$ being

$$
u(\xi)=(1+p \xi)^{-1-a} \exp \left[-\xi+p^{-1} \log (1+p \xi)\right] .
$$

(ii) The function $V$ converges for 


$$
\operatorname{ord} x< \begin{cases}\frac{1}{2}(1+1 /(p-1)), & p \geqslant 5, \\ \frac{1}{2}+\frac{1}{6}, & p=3, \\ \frac{1}{2}, & p=2 .\end{cases}
$$

The function $V^{-1}$ is holomorphic (i.e. $V$ is nonzero) for

$$
\text { ord } x<\varepsilon_{p}
$$

where

$$
\varepsilon_{p}= \begin{cases}\frac{1}{2}(1-1 /(p-1)), & p \geqslant 5 \\ \frac{1}{6}, & p=3\end{cases}
$$

and $\varepsilon_{2}>0$.

(iii) $V$ cannot be continued analytically into the region

$$
\left\{x \mid \operatorname{ord} x>\frac{1}{2}+2 /(p-1)\right\} \text {. }
$$

Proof. Assertion (i) is purely algebraic and may be checked by direct substitution. An alternate method is to restrict our attention to the case in which $a$ is real and positive. The differential equation satisfied by $\phi(a, c, x)$ has [6, p. 255] a solution valid for $\operatorname{Re} x>0, \operatorname{Re} a>0$,

$$
\psi(a, c ; x)=\frac{1}{\Gamma(a)} \int_{0}^{\infty} e^{-x \xi} \xi^{a}(1+\xi)^{c-a-1} d \xi / \xi .
$$

Replacing $x$ by $x / p$ and $\xi$ by $p \xi$ gives

$$
\psi\left(a, c ; \frac{x}{p}\right)=\frac{p^{a}}{\Gamma(a)} \int_{0}^{\infty} e^{-x \xi} \xi^{a}(1+p \xi)^{c-a-1} d \xi / \xi
$$

and thus for $\operatorname{Re} x<1, c=1 / p$,

$$
\psi\left(a, c ; \frac{1-x}{p}\right)=\frac{p^{a}}{\Gamma(a)} \int_{0}^{\infty} e^{-x(-\xi)} \xi^{a} u(\xi) d \xi / \xi .
$$

The asserted formula then follows by replacing $u$ by its power series expansion and using (say for $x$ real negative)

$$
\frac{1}{\Gamma(a)} \int_{0}^{\infty} e^{-x(-\xi)} \xi^{a+m} d \xi / \xi=\frac{1}{(-x)^{a+m}} \frac{\Gamma(a+m)}{\Gamma(a)} .
$$

Since the assertion is algebraic we may now ignore explicit and implicit hypotheses concerning the real parts of $a$ and $x$.

(ii) The domain of convergence of $V$ is deduced from the formula 


$$
-\xi+\frac{1}{p} \log (1+p \xi)=\sum_{j=2}^{\infty} \frac{(-1)^{j-1}}{p} \frac{(p \xi)^{j}}{j}
$$

which converges for ord $\xi>-1$. This shows that the argument in the exponential function in (7.6) has ordinal exceeding $1 /(p-1)$ if

$$
\text { ord } \xi> \begin{cases}-\varepsilon_{p}, & p \neq 2, \\ \frac{1}{2}, & p=2,\end{cases}
$$

and thus $u(\xi)$ converges and is bounded by 1 in this region. Thus by the Cauchy inequality

$$
\frac{1}{m} \text { ord } B_{m} \geqslant \begin{cases}\varepsilon_{p}, & p \neq 2, \\ -\frac{1}{2}, & p=2,\end{cases}
$$

which demonstrates $(7.8)$ for $p \neq 2$.

Since $(a)_{m} / m !=(-1)^{m}\left(\begin{array}{c}-a \\ m\end{array}\right)$ and since the binomial coefficient lies in $\mathbf{Z}_{p}$, we conclude that

$$
\operatorname{ord}(a)_{m} \geqslant m /(p-1)+O(\log m) .
$$

Equation (7.7) follows from this estimate and (7.13).

To verify (7.8) for $p=2$ we use an argument which could also be used to prove a slightly weaker form of (7.8) for all $p$. Hence there will be no reference to any particular value for $p$. The image of $x^{-1} L_{a}$ in $\bar{\Re}_{0}$ is $D+(\bar{a} / x)$ and hence by Theorem 6.2.3, this first order operator lifts uniquely to an element $M$

$$
M=D-\eta
$$

which is a one sided divisor of $x^{-1} L_{a}$ in $\Re_{A}$ where $A$ is a superadmissible subset of $\Omega \cup\{\infty\}$ containing all residue classes (including $\infty$ ) except for the zero class. The differential equation

$$
d y / d x=\eta y
$$

has at worst a regular singularity at infinity and hence there exists a formal solution of $M$ in $x^{\alpha} \Omega[[1 / x]]$ for some constant $\alpha$. This solution is of necessity a formal solution of $L_{a}$. The indicial polynomial of $L_{a}$ at infinity is of degree one and hence (7.5) gives a solution of (7.15). Thus $V^{\prime} / V$ is holomorphic in a disk $\left\{x \mid \operatorname{ord} x<\varepsilon_{p}\right\}$, where $\varepsilon_{p}>0$ is chosen so that the disk lies in the set $A$. This completes the proof of (7.8) for $p=2$, explicit estimates for $\varepsilon_{p}$ being given by equation (7.13) for $p \neq 2$.

(iii) By the method of Clark [2], the power series solutions for $L_{a}$ at $x=0$ converge for ord $x>\frac{1}{2}+2 /(p-1)$. If assertion (iii) were false then $x^{a}$ would 
be a meromorphic function on an annulus with center at the origin. This contradiction completes the proof of the proposition.

We impose on $\beta$ in (7.2) the condition that

$$
b>\beta>\left|p^{\varepsilon}\right|
$$

Thus

$$
M=D-\frac{V^{\prime}}{V}+\frac{a}{x}=V \circ\left(D+\frac{a}{x}\right) \circ \frac{1}{V}
$$

lies in $\Re_{C}$ as well as in $\Re_{C^{\prime}}$ and is stable on both $\mathscr{Q}_{C}$ and on $\mathbb{Q}_{C^{\prime}}^{*}$ (but not on $\left.\mathbb{Q}_{0}^{b}\right)$.

7.19 LEMMA. Let

$$
\lambda_{+}=\liminf _{m \rightarrow+\infty}|a+m|^{1 / m}, \quad \lambda_{-}=\liminf _{m \rightarrow+\infty}|a-m|^{1 / m},
$$

in both cases the limit being over $m \in \mathbf{N}$. If

$$
\lambda_{+}<1, \quad \lambda_{-}=1,
$$

then $M$ has index on $\mathbb{Q}_{C^{\prime}}^{*}$ but does not have index on $\mathbb{Q}_{C}$.

Proof. Multiplication by $V$ is an automorphism of both $\mathbb{Q}_{C}$ and of $\mathbb{Q}_{C^{\prime}}^{*}$ and hence by (7.18) the index of $M$ on these spaces is the same as that of $D+a / x$. As shown in $[11, \S 4.19]$ the index of $x D+a$ on $\mathbb{Q}_{C^{\prime}}^{*}$ (resp. $\left.\mathbb{Q}_{0}^{b}\right)$ is independent of the radius and depends only on $\lambda_{-}$(resp. $\lambda_{+}$). Thus by (7.20), $x D+a$ has index on $\mathbb{Q}_{C^{\prime}}^{*}$ but not on $\mathbb{Q}_{0}^{b}$ and hence by (7.3) $x D+a$ does not have index on $\mathbb{Q}_{C}$ as asserted. This completes the proof of the lemma.

Replacing $x^{-a}$ by an appropriate branch at $x=t$, equation (7.5) represents the unique solution of $L_{a}$ in $\mathbb{Q}_{t}$ and so

$$
L_{a}=N \cdot M
$$

where $N$ is injective on $\mathbb{Q}_{t}$. Thus Proposition 7.4 gives an illustration of Theorem 4.1. Explicitly

$$
N=p(1-x) D-x-p(1-x)\left(a / x-V^{\prime} / V\right)
$$

which shows that $N$ is stable on $\mathscr{Q}_{C}$. While $N$ is not stable on $\mathbb{Q}_{C^{\prime}}^{*}, N / x$ is stable on both $\mathbb{Q}_{C}$ and $\mathbb{Q}_{C^{\prime}}^{*}$ and is injective on $\mathbb{Q}_{t}$. Thus by $\S 4.3$ (with $a$ fixed) $N / x$ has index on both $Q_{C}$ and $\mathbb{Q}_{C^{\prime}}^{*}$ for all $b, \beta$ close enough to 1 .

Let now $a$ be chosen satisfying (7.20), let $b=1$, let $\beta$ satisfy (7.17) and be close enough to but strictly less than 1 . Then by $7.19, L_{a} / x$ has index on $\mathbb{Q}_{C^{\prime}}^{*}$ but not on $\mathbb{Q}_{C}$ and hence $L_{a}$ has index on the first but not on the second. We conclude: $L_{a}$ does not have index on $Q_{0}$. Furthermore for each $b$ real and 
close to unity $L_{a}$ fails to have index on $\mathbb{Q}_{0}^{b}$. By the same methods we can show that $\mathfrak{Q}_{0} \cap L_{a} \mathfrak{Q}_{0}^{b}$ has infinite codimension in $\mathfrak{Q}_{0}$ for all $b<1$ such that $b$ is close enough to unity.

It only remains to verify that there exists $a \in \mathbf{Z}_{p}$ satisfying (7.20). As in [7] we put $p_{1}=1, p_{n+1}=p_{n}+p^{p_{n}}$ for all $n \geqslant 1$ and let $a=-\lim _{n} p_{n}$. Here $\lambda_{+}=1 / p$. We compute $a=\sum_{i=0}^{\infty} y_{1} p^{i}$ where

$$
\begin{aligned}
y_{i} & =p-2 & & \text { if } i=p_{n} \text { for some } n \geqslant 2, \\
& =p-1 & & \text { if otherwise, }
\end{aligned}
$$

and so if $m \in \mathbf{N}, m<p^{s}$, then $\operatorname{ord}(a-m) \leqslant s+1$, i.e. $\operatorname{ord}(a-m)$ $=O(\log m)$. This shows that $\lambda_{-}=1$.

7.21 In the construction of the above example we could have discarded the demonstration of holomorphy of $1 / V$ (equation (7.8)).

It follows from (7.7) that if $\gamma_{0} \in(|\sqrt{p}|, 1)$ then $V$ is holomorphic in the complement of $D\left(0, \gamma_{0}^{-}\right)$and hence there exists a polynomial $P$, such that $V / P$ is analytic element without zeros on that complement. Let $n=1+\operatorname{degree} P$. We define $L^{\prime}$ by

$$
L^{\prime}=\left(1 / x^{n}\right) \circ L_{a} \circ P=N^{\prime} \circ M^{\prime}
$$

where

$$
N^{\prime}=\frac{1}{x^{n}} \circ N \circ P, \quad M^{\prime}=\frac{1}{P} \circ M \circ P=\frac{V}{P} \circ\left(D+\frac{a}{x}\right) \circ \frac{P}{V} .
$$

Thus for $\beta \geqslant \gamma_{0}$ we may conclude that $N^{\prime}$ and $M^{\prime}$ are stable on both $\mathscr{Q}_{C}$ and on $Q_{C^{\prime}}^{*}$ and the proof of Lemma 7.19 shows that under condition (7.20), $M^{\prime}$ has index on $\mathbb{Q}_{C^{\prime}}^{*}$ but does not have index on $\mathfrak{Q}_{C}$. Thus if $a$ satisfies condition (7.20) and $b=1$ and $\beta$ is strictly less than but sufficiently close to 1 then $L^{\prime}$ also has index on $\mathbb{Q}_{C^{\prime}}^{*}$ but not on $\mathbb{Q}_{C}$ and hence the same holds for $L_{a}$. This shows that $L_{a}$ does not have index as endomorphism of $\mathfrak{Q}_{0}$.

7.22 Modifying slightly the notation of Clark [2], we say that $a \in \mathbf{Z}_{p}$ is nonLiouville if

$$
\operatorname{ord}(a-n)=O(\log |n|)
$$

as $n \rightarrow \pm \infty$ in $\mathbf{Z}$. (Here $|n|$ and $\log$ are in the archimedean sense.)

In our example $L_{a}$ has exponent at infinity which fails to satisfy this condition. We therefore ask if a counterexample to [11, §4.27] may be constructed with rational function coefficients and non-Liouville exponents.

7.23 E. Calabi has observed that the operator

$$
L_{a, \nu}=p^{\nu}(1-x) D^{2}-X D-a
$$


with $\nu$ integral, $\nu>1$, could also be used to demonstrate the nonexistence of index. The advantage is that with large values of $\nu$ the computations corresponding to Proposition 7.4. are greatly simplified.

8. Galois theory of $\Omega$ over $K$. Let $\Omega$ be an algebraically closed field of characteristic zero which is maximally complete (and hence complete) under a rank one valuation. Let $K$ be a complete subfield and let $R$ be the closure in $\Omega$ of the algebraic closure of $K$. Let $G$ be the group of continuous automorphisms of $\Omega$ over $K$ and let $G_{K}$ be the continuous automorphisms of $K$ over $K$.

By a well-known theorem of Tate (extended to the nondiscrete case by Ax [1]):

The elements of $\hat{K}$ fixed under all elements of $G_{K}$ are precisely the elements of $K$.

We demonstrate a related result.

THEOREM. The elements of $\Omega$ fixed under all elements of $G$ are precisely the elements of $K$.

Note. We do not know if this result is valid without the hypothesis that $\Omega$ is maximally complete. Thus this theorem does not imply the result of Tate. Furthermore Tate's theorem is used in the proof of Lemma 8.1 below and so there is no assertion of a new proof of that result.

We shall need several preliminary results.

8.1 Lemma. Let $\alpha$ be an element of $\Omega$ not in $K$. There exists a continuous automorphism $\sigma$ of $\hat{K}(\alpha)$ which leaves $K$ pointwise fixed but does not leave $\alpha$ fixed.

Proof. If $\alpha$ lies in $\hat{K}$, then this assertion is equivalent to Tate's theorem. Thus, we may assume that $\alpha \notin \hat{K}$ and hence the distance of $\alpha$ from $\hat{K}, d(\alpha, \hat{K})=\gamma>0$. Since $\alpha$ is transcendental over $\hat{K}$ we may for each $b \in \hat{K}$ define an automorphism, $\sigma_{b}$, of $\hat{K}(\alpha)$ over $\hat{K}$ by setting $\sigma_{b} \alpha=\alpha+b$. To complete the proof it is enough to show that $\sigma_{b}$ is continuous for some $b \neq 0$. We assert that this is the case if $|b| \leqslant \gamma$. We note that $\sigma_{b}$ is continuous if and only if it is an isometry of $\hat{K}(\alpha)$. It is enough to consider the action of $\sigma_{b}$ on $\hat{K}[\alpha]$ and since $\hat{K}$ is algebraically closed it is enough to show that

$$
|\alpha+a+b|=|\alpha+a|
$$

for all $a \in \hat{K}$. By hypothesis

$$
|\alpha+a| \geqslant \gamma \geqslant|b|, \quad|\alpha+(a+b)| \geqslant \gamma \geqslant|b|,
$$

and so (8.1.1) follows since the valuation is ultrametric.

Theorem 8 is a direct consequence of Lemma 8.1 and the following lemma.

8.2 Lemma. Let $\Omega$ be maximally complete, let $K$ be a closed subfield. Then each 
continuous automorphism of $K$ may be extended to a continuous automorphism of $\Omega$.

This lemma is the consequence of the following slight generalization which is better adapted to transfinite induction arguments.

8.3 Lemma. Let $\Omega$ and $K$ be as above. Let $\tau$ be a continuous isomorphism of $K$ into $\Omega$. (Of course, $K$ and its image $K^{\tau}$ have residue class fields which are subfields of $\bar{\Omega}$, the residue class field of $\Omega$.) Suppose that the residue class fields of $K$ and of $K^{\tau}$ are equal. Then $\tau$ may be extended to a continuous automorphism of $\Omega$.

Proof. We consider pairs, $(\sigma, L)$, of fields, $L$, intermediate between $K$ and $\Omega$ and continuous automorphisms, $\sigma$, of $L$ into $\Omega$ such that $L$ and $L^{\sigma}$ have the same residue class fields. Such pairs are ordered in the obvious way and by Zorn's lemma there exists a maximal element $(\sigma, L)$ such that $(\sigma, L)>(\tau, K)$.

By a lengthy but conventional argument, we will show that $L=\Omega=L^{\sigma}$. There are several steps.

8.3.1 $L$ is complete (by maximality).

8.3.2 We assert that $L$ is algebraically closed. If $z$ is algebraic over $L$ then $\sigma$ may be extended to an isomorphism of $L(z)$ into $\Omega$ which must be an isometry since the valuation of $L(z)$ which extends the given valuation of $L$ is unique. Thus, by another application of Zorn's lemma, $\sigma$ may be extended to a continuous isomorphism, $\hat{\sigma}$, of $\hat{L}$ into $\Omega$. The residue class field of $\hat{L}$ is the algebraic closure in $\bar{\Omega}$ of $\bar{L}\left(=\overline{L^{\circ}}\right)$ and hence is the same as the residue class field of $\hat{L}^{\hat{\sigma}}$. This completes the proof since $(\sigma, L)$ is maximal.

8.3.3 We assert that $L$ and $\Omega$ have the same value group. Otherwise there exists $Y \in \Omega$ such that $|Y|$ does not lie in the value group of $L$ (which is the same as the value group of $L^{\sigma}$ ). Then $Y$ is transcendental over both $L$ and $L^{\sigma}$. We may thus extend $\sigma$ to an isomorphism of $L(Y)$ with $L^{\sigma}(Y)$ by setting $\sigma Y=Y$. For $\beta \in L$ we have $|Y| \neq|\beta|=\left|\beta^{\sigma}\right|$ and hence

$$
|Y-\beta|=\operatorname{Sup}(|Y|,|\beta|)=\left|Y-\beta^{\sigma}\right|
$$

and then the extension of $\sigma$ is isometric and hence continuous. Trivially $L(Y)$ has the same residue class field as $L$ and hence $L(Y)$ and $L^{\sigma}(Y)$ have the same residue class fields. This contradicts the maximality of $(\sigma, L)$ and completes the proof of 8.3.3.

8.3.4 We assert that $L$ and $\Omega$ have the same residue class field. Otherwise there exists $Y \in \Omega,|Y|=1$ such that $\bar{Y} \notin \bar{L}=\overline{L^{\sigma}}$. Then, $\bar{Y}$ is transcendental over $\bar{L}$. By putting $\sigma(Y)=Y$ we extend $\sigma$ to a continuous isomorphism of $L(Y)$ into $L^{\sigma}(Y)$ and the residue class fields coincide with $\bar{L}(\bar{Y})$ and are thus identical. This contradicts the maximality of $(\sigma, L)$.

8.3.5 We assert that $L$ is maximally complete. Suppose otherwise and let 
$\left\{D\left(a_{i}, \gamma_{i}\right)\right\}_{i \in \mathbf{N}}$ be a sequence of imbedded disks with centers in $L$ and no common point in $L$. Since $\Omega$ is maximally complete, let $x$ be a common point in $\Omega$. Clearly $x$ is transcendental over $L$.

We observe that $\left\{D\left(\sigma a_{i}, \gamma_{i}\right)\right\}_{i \in \mathrm{N}}$ is again a sequence of imbedded disks and hence has a common point, $y$, in $\Omega$. This point cannot lie in $L^{\sigma}$ as otherwise $\sigma^{-1} y$ would lie in $L \cap D\left(a_{i}, \gamma_{i}\right)$ for each $i \in \mathrm{N}$ which is impossible. Since $L^{\sigma}$ is also algebraically closed, $\sigma$ may be extended to an isomorphism of $L(x)$ with $L^{\sigma}(y)$ by setting $\sigma x=y$. To verify continuity it is enough to show that

$$
|x-\alpha|=|y-\sigma \alpha|
$$

for all $\alpha \in L$. We know that

$$
|x-\alpha|>d(x, L)=\inf \gamma_{i}
$$

and hence there exists $i \in \mathbf{N}$ such that $\left|x-a_{i}\right| \leqslant \gamma_{i}<|x-\alpha|$. Hence $|x-\alpha|=\left|\alpha-a_{i}\right|>\gamma_{i}$ but $y \in D\left(\sigma a_{i}, \gamma_{i}\right)$ and so

$$
|y-\sigma \alpha|=\left|\left(y-\sigma a_{i}\right)+\sigma\left(a_{i}-\alpha\right)\right|=\left|a_{i}-\alpha\right|=|x-\alpha|,
$$

which completes the verification of (8.3.5.1). Furthermore, $\bar{L}=\overline{L^{\sigma}}=\bar{\Omega}$ shows that $L(x)$ and $L^{\sigma}(y)$ have the same residue class field. This contradicts the maximality of $(\sigma, L)$.

We have shown that $L$ (resp. $L^{\sigma}$ ) has the same value group and the same residue class field as $\Omega$. Thus $\Omega$ is an immediate extension of $L$ (resp. $L^{\sigma}$ ). Furthermore, $L$ (resp. $L^{\sigma}$ ) is maximally complete. Hence [14, Chapter 2] $L=\Omega=L^{\sigma}$. This completes the proof of Lemma 8.3.

Proof of Theorem 8. Let $\alpha$ be element of $\Omega$ not in $K$. Let $\sigma$ be the continuous automorphism of $\hat{K}(\alpha)$ over $K$ whose existence is assured by Lemma 8.1. This automorphism may be extended by continuity to the completion of $\widehat{R}(\alpha)$ and by Lemma 8.2 to a continuous automorphism of $\Omega$. This completes the proof of the theorem.

8.4 As preparation for the application of the above theorem, we consider the orbit of a disk under the action of $G$.

Lemma. If a disk $T=D\left(\alpha, r^{-}\right)$contains no element of $\hat{K}$, then $T$ has an infinite set of distinct images under the action of $G$.

Proof. Let $\gamma=d(\alpha, \hat{K})$. Clearly, $\gamma \geqslant r$. We consider two possibilities:

8.4.1 Case 1. Either the real number $r$ lies in the value group of $\hat{K}$ or $\gamma>r$.

We know from the proof of Lemma 8.1 that if $b \in \hat{K},|b| \leqslant \gamma$, then $\sigma_{b}: \alpha \mapsto \alpha+b$ defines a continuous automorphism of $\hat{K}(\alpha)$, which by Lemma 8.2 can be extended to an element of $G$ (which we will denote by the same symbol). The image $\sigma_{b} T$ of $T$ is in one to one correspondence with the class 
of $b$ modulo $D\left(0, r^{-}\right)$which demonstrates the lemma in this case.

8.4.2 Case 2. $r$ does not lie in the value group of $\hat{K}$, and $\gamma=r$.

If $\beta_{1}$ and $\beta_{2}$ are elements of $D\left(\alpha, r^{+}\right)$whose difference does not lie in $D\left(0, r^{-}\right)$, then at most one of the two disks $D\left(\beta_{i}, r^{-}\right)(i=1,2)$ can contain an element of $\hat{K}$ (as otherwise $r=\left|\beta_{1}-\beta_{2}\right|$ would lie in the value group of $\hat{K}$ ). Thus there exist an infinite set of distinct disks $D\left(\beta, r^{-}\right)$which lie in $D\left(\alpha, r^{+}\right)$ and contain no element of $\hat{K}$. Such a $\beta$ must also be transcendental over $\hat{K}$ and hence $\alpha \mapsto \beta$ defines an isomorphism, $\sigma$, of $\hat{K}(\alpha)$ with $\hat{K}(\beta)$.

8.4.2.1 We assert that $\sigma$ is continuous. That is we assert that for each $c \in \hat{K}$ we have $|\alpha-c|=|\beta-c|$. This follows from the relations

$$
|\alpha-c| \geqslant r,|\beta-c| \geqslant r, \quad|\alpha-\beta|=r
$$

if we consider the two possibilities, $|\alpha-c|>r$ and $|\alpha-c|=r$. This completes the verification of 8.4.2.1. To be able to apply Lemma 8.3, we must show that $\hat{K}(\alpha)$ and $\hat{K}(\beta)$ have the same residue class fields.

8.4.2.2 We assert that $\hat{K}(\alpha)($ resp. $\hat{K}(\beta))$ has the same residue class field as $R$. Here again we consider two possibilities.

8.4.2.2.1 The value of $d(\alpha, \hat{K})$ is assumed. Thus there exists $c \in \hat{K}$ such that $|c-\alpha|=r$. Thus replacing $\alpha$ by $\alpha-c$ we may assume that $|\alpha|=r$. If $f$ is any element of $\hat{K}[X]$ then by hypothesis 8.4.2 the newton polygon of $f$ has no side of slope corresponding to $r$ and that a line of support of that slope which passes through the origin can have no contact with the polygon except at the origin itself. This shows that if $f(\alpha)$ is a unit, then it is in the same residue class as $f(0)$. This completes the proof of 8.4.2.2 in this case.

8.4.2.2.2 The value of $d(\alpha, \hat{K})$ is not assumed. In this case $\alpha$ is pseudolimit of a transcendental pseudo convergent sequence of $\hat{K}$ and hence [14, Chapter 2] lies in an immediate extension of $\hat{K}$. This completes the proof of 8.4.2.2.

It now follows from Lemma 8.3 that $\sigma$ may be extended to an element of $G$ and clearly $\sigma D\left(\alpha, r^{-}\right)=D\left(\beta, r^{-}\right)$. This completes the treatment of 8.4.2 and hence of the lemma.

8.5 Application. Let $A$ be an infra connected subset of $\Omega$ which is stable under $G$. Let $H_{K}(A)$ (resp. $\left.H_{\Omega}(A)\right)$ be the set of analytic elements of $A$ defined over $K$ (resp. $\Omega$ ), i.e. uniform limits on $A$ of elements of $K(X)$ (resp. $\Omega(X)$ ), having no poles in $A$. The group $G$ operates on $H_{\Omega}(A)$ by $H_{\Omega}(A) \ni f \mapsto f^{\sigma}$ where

$$
f^{\sigma}(\sigma x)=\sigma(f(x))
$$

for all $x \in A$.

THEOREM. The elements of $H_{\Omega}(A)$ fixed under $G$ are precisely the elements of $H_{K}(A)$. 
Proof. Let $f$ be an element of $H_{\Omega}(T)$ and let $\mathcal{T}$ be the set of all trous of the set $A$. By the generalized Mittag-Leffler Theorem [10],

$$
f=\sum_{T \in \mathscr{T}} f_{T},
$$

a unique decomposition into elements $f_{T} \in H_{\Omega}(\mathbf{C} T)$ such that $f_{T}$ vanishes at infinity (if $\infty \notin T$ ) and such that $f-f_{T}$ extends analytically to $A \cup T$. If $\sigma \in G$, then

$$
\left(f_{T}\right)^{\sigma}=\left(f^{\sigma}\right)_{T^{\sigma}}
$$

and hence if $f=f^{\sigma}$ then

$$
\left(f_{T}\right)^{\sigma}=f_{T^{\circ}}
$$

for all $T \in \mathcal{T}$.

We now consider an element $f$ of $H_{\Omega}(A)$ which is invariant under $G$. We assert that $f_{T} \neq 0$ implies

$$
T \cap \hat{K} \neq \varnothing .
$$

Indeed, $f_{T} \neq 0$ implies $\left\|f_{T}\right\|_{\mathbf{C} T}=a>0$ and hence by equation (8.5.4), $\left\|f_{T^{*}}\right\|_{C^{*}}=a$ for all $\sigma \in G$.

Thus $T$ can have only a finite set of distinct conjugates under the action of $G$ and hence (8.5.5) is a consequence of Lemma 8.4. Thus if $f_{T} \neq 0$ we may put $T=D\left(\alpha, r^{-}\right)$with $\alpha$ algebraic over $K$. Let then $M$ be a finite galois extension of $K$ which contains $\alpha$. We may write

$$
f_{T}=\sum_{j=1}^{\infty} A_{j} /(X-\alpha)^{j}
$$

with $A_{j} \in \Omega$ for all $j$ and

$$
\left|A_{j}\right| \rightarrow 0 \text { as } j \rightarrow \infty .
$$

However, $T$ is stable under the group, $G_{M}$, of continuous automorphisms of $\Omega$ over $M$. Thus by (8.5.4), $f_{T}$ is invariant under $G_{M}$ and thus by Theorem 8, each $A_{j}$ lies in $M$. It follows from (8.5.7) that $f_{T}$ is uniform limit on $A$ of elements of $M(X)$ having pole only at $\alpha$.

We have shown that the galois group \&s of $M$ over $K$ operates on $f_{T}$. Let $\mathfrak{G}=\{\sigma \in \mathbb{S}|| \sigma \alpha-\alpha \mid<r\}$. Clearly $\mathfrak{\mathscr { E }}$ is a subgroup of $\mathbb{B S}$ and for $\sigma, \tau \in \mathbb{S}$ we have $\left(f_{T}\right)^{\sigma}=\left(f_{T}\right)^{\tau}$ if and only if $\sigma^{-1} \tau \in \mathfrak{Q}$. Thus let

$$
\text { (S) }=\bigcup_{i=1}^{S} \sigma_{i} \mathfrak{B}
$$


be the coset decomposition of $\mathbb{E S}$ corresponding to $\mathfrak{S}$. Then the full set of conjugates of $T$ under $G$ is $\left\{T_{i}\right\}_{i=1}^{s}$, where for each $i(=1,2, \ldots, s) T_{i}=$ $D\left(\sigma_{i}, \alpha, r^{-}\right)$. Thus in the Mittag-Leffler decomposition of $f$, the component corresponding to $T$ and its conjugates is precisely

$$
\sum_{i=1}^{s} f_{T_{i}}=\sum_{i=1}^{s}\left(f_{T}\right)^{\sigma_{i}}=h^{-1} \sum_{\sigma \in \mathcal{S}}\left(f_{T}\right)^{\sigma}
$$

where $h=\operatorname{card}(\mathfrak{E})$. The left side of this equation may be written as

$$
\lim _{n \rightarrow \infty} h^{-1} S_{M / K} \sum_{j=1}^{n} A_{j} /(X-\alpha)^{j}
$$

where $S_{M / K}$ is the mapping of $M(X)$ onto $K(X)$ which extends in the obvious way the trace mapping of $M$ into $K$. It is now easy to verify that $f$ lies in $H_{K}(A)$ as asserted.

\section{Problems and special results.}

9.1 Supersingularity. Let $A$ be a standard set. Let $L \in \Re_{A}$ have kernel at $t$ which lies in $Q_{t}$. Then by 4.2 .7 for each disk $D\left(a, 1^{-}\right)$in $A(|a| \leqslant 1)$ in which $L$ has at worst removable singularities, we may associate a newton polygon as outlined in an earlier article [3]. Namely for each positive real number $\beta$ let $g_{a}(\beta)$ be the dimension of the kernel of $L$ in $W_{a}^{1, \beta}$. Let $\beta_{1}, \ldots, \beta_{s}$ be the points of discontinuity of $g_{a}\left(\beta_{1}=0\right.$ if $\left.g_{a}(0)>0\right)$ and let $c_{i}$ be the jump of $g_{a}$ at $\beta_{i}(i=1,2, \ldots, s)$. The newton polygon is constructed so that the $j$ th side has slope $\beta_{j}$ with projected length $c_{j}$.

9.1.1 Conjecture [4]. The polygon for $L$ at $t$ lies on or below the polygon for $L$ at any other point.

A special case of this conjecture is given by Theorem 2.4 above.

9.1.2 Definition. We say that $L$ is supersingular at those residue classes for which the newton polygon of $L$ does not coincide with the polygon at $t$.

9.1.3 CONJECTURE. $L$ is supersingular at only a finite number of residue classes in $A$.

In particular [4],

9.1.4 CONJECTURE. In (2.4.1) (above) equality holds at all but a finite number of residue classes in $A$.

While known for many cases $([11, \S 4.26 .2])$ this conjecture is still open for operators of order 2 with rational coefficients. By Theorem 2.4 this conjecture is equivalent to the following conjecture.

9.1.5 Conjecture. If $A$ is standard set, $L \in \Re_{A}, M, N \in \Re$, and $L$ $=N \cdot M$ then $M \in \Re_{B}$ for some admissible subset $B$ of $A$.

This conjecture is implied by Conjecture 3.1.5 above.

9.2 A special case of Conjecture 3.1.5 is the linear case. Thus 
9.2.1 Conjecture. Let $A$ be a standard set, $L \in \Re_{A}$; let $u \in E, L u=0$, then $u \in H(B)$ for some admissible subset $B$ of $A$.

For the case in which $L \in \Re_{0}$, [13], Conjecture 9.2.1 is now known to be a direct consequence of the Mittag-Leffler theorem and indeed the same conclusion holds if $L u=v, L \in \Re_{0}, u \in E$ and $v \in H(A)$.

Without the hypothesis $L \in \Re_{0}$, Conjecture 9.2.1 has been verified only in the case of order one. This was done by Motzkin [7] by means of his theory of singular factors.

Note that in 9.2.1 it may be assumed that $L$ has a full set of solutions in $\mathbb{Q}_{t}$ as if $M, N$ are defined by equation (4.2.1) then $M u$ lies in $W_{t}^{1,0}$ and hence must be zero as $N$ has no solutions in that space.

9.3 We now give an application of Conjecture 9.2.1. We assume $L \in \Re_{0}$ since the conjecture is known in that case.

LEMMA. Let $L \in \Re_{0}$ be of order 2 and have Riccati equation with two distinct solutions in $E$. Then these two solutions lie in $E_{\mathrm{ad}}$.

Proof. Let $L=D^{2}+a D+b$ and let $\eta_{1}, \eta_{2}$ be distinct solutions in $E$ of the Riccati equation

$$
\eta^{2}+\eta^{\prime}+a \eta+b=0
$$

Now let $u_{1}, u_{2}$ be germs of analytic functions at $t$ such that

$$
u_{i}^{\prime} / u_{i}=\eta_{i}, \quad i=1,2 .
$$

Thus $u_{1}, u_{2}$ are independent elements of $\operatorname{ker}_{t} L$. Thus

$$
\eta_{1}-\eta_{2}=w / u_{1} u_{2}
$$

where $w$ is the wronskian of $L$. We observe that $u_{1} u_{2}$ is a solution of the third order linear differential equation (in $\Re_{0}$ ) whose other two solutions are $u_{1}^{2}, u_{2}^{2}$. Furthermore twisting this third order linear differential operator by $w$, we obtain $N$, a third order linear differential operator, $N \in \Re_{0}$ such that $u_{1} u_{2} / w$ lies in $\operatorname{ker}_{t} N$. By (9.3.3), $1 /\left(\eta_{1}-\eta_{2}\right) \in E \cap \operatorname{ker}_{t} N$ and therefore by [13], we conclude that

$$
\eta_{1}-\eta_{2} \in E_{\mathrm{ad}}
$$

The Riccati equation shows that

$$
\left(\eta_{1}-\eta_{2}\right)\left(\eta_{1}+\eta_{2}\right)+\left(\eta_{1}-\eta_{2}\right)^{\prime}+a\left(\eta_{1}-\eta_{2}\right)=0
$$

and hence $\eta_{1}+\eta_{2} \in E_{\text {ad }}$. Then $\eta_{1}$ and $\eta_{2} \in E_{\text {ad }}$ as asserted. 


\section{REFERENCES}

1. J. Ax, Zeros of polynomials over local fields-The Galois action, J. Algebra 15 (1970), 417-428. MR 41 \# 8386.

2. D. N. Clark, $A$ note on the p-adic convergence of solutions of linear differential equations, Proc. Amer. Math. Soc. 17 (1966), 262-269. MR 32 \# 4350.

3. B. Dwork, On p-adic differential equations. II, Ann. of Math. (2) 98 (1973), 366-376.

4. - On p-adic differential equations. III, Invent. Math. 20 (1973), 34-45.

5. A. Erdélyi, Uber die Integration der Whittakerschen Differentialgleichung in geschlossener Form, Monatsh. Math. Phys. 46 (1937), 1-9.

6. A. Erdélyi, W. Magnus, F. Oberhettinger and F. G. Tricomi, Higher transcendental functions, Vol. I, McGraw-Hill, New York, 1953. MR 15, 419.

7. E. Motzkin, La décomposition d'un élément analytique en facteurs singuliers. Ann. Inst. Fourier (Grenoble) 27-I (1977), 67-82.

8. - Sur des ensembles analytiques p-adiques définis à l'aide de fonctions analytiques bornées, Groupe d'Étude d'Analyse Ultramétrique (Amice, Robba), 1975/76.

9. E. Poole, Introduction to the theory of linear differential equations, Oxford, 1936.

10. P. Robba, Fonctions analytiques sur les corps valués ultramétriques complets, Prolongement Analytique et Algèbres de Banach Ultramétriques, Astérique, No. 10, Soc. Math. France, Paris, 1973, pp. 109-218, 219-220. MR 50 \# 10307.

11. - On the index of p-adic differential operators. I, Ann. of Math. (2) 101 (1975), 280-316. MR 51 \#498.

12. _- On the index of p-adic differential operators. II, Duke Math. J. 43 (1976), 19-31.

13. - Prolongement des solutions d'une équation différentielle p-adique, C. R. Acad. Sci. Paris A 279 (1974), 153-154. MR 50 \#2136.

14. O. F. G. Schilling, The theory of valuations, Math. Surveys, no. 4, Amer. Math. Soc., Providence, R.I., 1950. MR 13, 315.

15. M. van der Put, The non-archimedean corona problem, Table Ronde d'Analyse Non Archimédienne (Paris, 1972), Bull. Soc. Math. France, Mém. No. 39-40, Soc. Math. France, Paris, 1974, pp. 287-317. MR 51 \#939.

Department of Mathematics, Princeton University, Princeton, New Jersey 08540

Département de Mathématique, Université de Paris XI Paris, 91400 Orsay, France 